\title{
DO WE NEED RETARDED DELIVERY OF BONE GROWTH FACTORS IN FACIAL BONE REPAIR? AN EXPERIMENTAL STUDY IN RATS
}

\author{
N. Moser ${ }^{1}$, N. Lohse ${ }^{2}$, J. Goldstein ${ }^{1}$, P. Kauffmann ${ }^{1}$, B. Sven ${ }^{3}$, M. Epple ${ }^{3}$ and H. Schliephake ${ }^{1, *}$ \\ ${ }^{1}$ Department of Oral and Maxillofacial Surgery, George-Augusta-University, 37075 Göttingen, Germany \\ ${ }^{2}$ Department of Oral and Maxillofacial Surgery, Klinikum Bremerhaven, 27574 Bremerhaven, Germany \\ ${ }^{3}$ Inorganic Chemistry and Centre for Nanointegration Duisburg-Essen (CeNIDE), University of \\ Duisburg-Essen, 45117 Essen, Germany
}

\begin{abstract}
The aim of the present study was to evaluate the effect of different dosages of retarded vs. rapid release of bone morphogenic protein 2 (BMP2) at different recipient sites. Porous composite poly(D,L-lactic acid) (PDLLA) $/ \mathrm{CaCO}_{3}$ scaffolds were loaded with three different dosages of rhBMP2 $(24 \mu \mathrm{g}, 48 \mu \mathrm{g}$ and $96 \mu \mathrm{g})$ and implanted, together with blank controls, both into non-healing defects of the mandibles and into the gluteal muscles of 24 adult male Wistar rats. After 26 weeks, bone formation and expression of bone specific markers [alkaline phosphatase (AP) and Runx2] were evaluated by histomorphometry and immunohistochemistry. Results showed that the mode of delivery had no quantitative effect on bone formation in mandibular sites. Expression of AP and Runx2 showed significant differences among the three dosage groups. There were significant correlations between the expression of both AP and Runx2, as well as, the extent of bone formation, with both retarded and rapid release of rhBMP2. In ectopic sites, retarded release significantly enhanced bone formation in the low and medium dosage groups, compared to rapid release. Expression of AP was significantly higher and Runx2 significantly lower in ectopic sites, compared to mandibular sites. Significant correlations between the expression of bone specific markers and bone formation occurred only in the retarded delivery groups, but not in the rapid release groups. Within the limitations of the experimental model, it was concluded that retarded delivery of BMP2 was effective, preferably in sites with low or nonexisting pristine osteogenic activity. Expression of bone specific markers indicated that osteogenic pathways might be different in mandibular vs. ectopic sites.
\end{abstract}

Keywords: Bone morphogenic protein, retarded release, ectopic, orthotopic, bone specific markers, bone formation.

*Address for correspondence: Prof. Dr Dr H. Schliephake, Department of Oral and Maxillofacial Surgery, George-Augusta-University, Robert-Koch-Str. 40, 37075 Göttingen, Germany.

Email: schliephake.henning@med.uni-goettingen.de

\section{Introduction}

Bone growth factors (BMPs), namely BMP2 and BMP7, have been in clinical use for several years with a variety of indications (Kelly et al., 2016; Delawi et al., 2016; Friedländer et al., 2001; Buttermann, 2008). Dosages applied are mostly excessive compared to physiological levels, with adverse events and serious side effects being reported in many studies (Burkus et al., 2002; Smucker et al., 2006, Hwang et al., 2009). One reason for the use of supraphysiological dosages is the insufficient mode of delivery, indicating the need for the development of controlled-release carriers to reduce the applied dosages. This has prompted extensive research efforts, with a large variety of materials being tested and validated (for review see Kanczler and Oreffo, 2008; Gothard et al., 2014).
Alongside this large body of research, experimental reports indicate that retarded release may be of little effect with regard to the amount of bone generated (Gruber et al., 2015; Geuze et al., 2012). In addition, the reaction to different release kinetics of bone growth factors may be site specific, with respect to orthotopic vs. ectopic sites (Kempen et al., 2009; Patel et al., 2008).

Reports on the clinical use of bone morphogenetic proteins in craniofacial repair suggest that, in human applications, recipient site reactions to BMPs vary depending on the nature of the defect. On the one hand, rapid delivery of BMP2 from collagen sponges for primary mandibular reconstructions after segmental resections of benign bone pathology with preserved periosteum is very successful (Clokie and Sandor, 2007; Herford et al., 2007; Carter et al., 2008; Herford and Cicciu, 2010; Glied and Kraut, 2010). 
However, BMP2 rapid delivery is associated with a high percentage of failure in sites of secondary reconstructions, after infection or previous surgery (Schliephake, 2015). If recipient site conditions in experimental animals are supposed to mimic clinical situations, orthotopic sites in adult animals may represent clinical defects with fresh bone wounds and viable periosteum. Instead, ectopic sites may reflect clinical situations with poor or even absent pristine osteogenic activity, such as orofacial defects after repeated surgical interventions.

Thus, it was felt desirable to analyse, in a systematic way, the effect of different dosages of BMP2, with different modes of delivery and under different recipient site conditions, to assess the usefulness of retarded delivery. Gas foamed porous poly(D,L-lactic acid) (PDLLA) $/ \mathrm{CaCO}_{3}$ composite carriers were used for both retarded and rapid delivery of rhBMP2. A rodent model was chosen, since it is recommended for the comparison of multiple groups requiring larger numbers of animals (Hollinger and Kleinschmidt, 1990). Observations were carried on for long period, corresponding to intervals of long-term bone regeneration in humans, as it is well-known that bone turnover is several times higher in rodents than in humans and that bone growth continues much longer after sexual maturity is reached in rats, compared to other animals (Histing et al., 2001; Garcia et al., 2013). Moreover, effect of bone growth factor delivery may be temporary both in negative and positive direction (Schliephake et al., 2015a, Hernandez et al., 2012; Ramazanoglu et al., 2013; Guillot et al., 2016). Therefore, late stages of bone healing appeared to be even more useful, when addressing sustained effects of retarded vs. rapid delivery of bone growth factors.

\section{Material and Methods}

\section{Preparation of delivery devices}

Porous carriers for the delivery of rhBMP2 were produced using gas foaming of PDLLA/CaCO composite granules, as previously described (Schliephake et al., 2015b). Briefly, amorphous PDLLA (16 g, RESOMER ${ }^{\circledR} \mathrm{R} 208$, inherent viscosity $1.8 \mathrm{dL} / \mathrm{g}$; Boehringer, Ingelheim, Germany) and $4 \mathrm{~g}$ of $\mathrm{CaCO}_{3}$ (average particle size $12 \mu \mathrm{m}$; Schaefer Kalk GmbH and Co. KG, Diez, Germany) were dry-processed into granules of 200-400 $\mu \mathrm{m}$ in diameter (Schliephake et al., 2015b). For rhBMP2 retarded delivery carriers, the resulting powder was mixed with aqueous solutions of rhBMP-2 (from Escherichia coli) (ReliaTech, Braunschweig, Germany) at concentrations of 400, 800 and $1600 \mu \mathrm{g} / \mathrm{g}$ of composite (groups 1A, 1B and $1 \mathrm{C}$, respectively). The mixtures of the different dosages of rhBMP2 and PDLLA-CaCO $\mathrm{C}_{3}$ powder were lyophilised overnight. Porous carriers of $8 \mathrm{~mm}$ diameter and $3 \mathrm{~mm}$ thickness were fabricated using supercritical carbon dioxide $\left[\mathrm{CO}_{2}\right.$ pressure of $10 \mathrm{MPa}$ for $2 \mathrm{~h}$ at $37^{\circ} \mathrm{C}$, filling time $20 \mathrm{~min}$, incubation time
$120 \mathrm{~min}$, venting time $20 \mathrm{~min}(500 \mathrm{kPa} / \mathrm{min})]$ to foam $0.06 \mathrm{~g}$ each of the growth factor-loaded granular powder, in custom-made polytetrafluorethylene (PTFE) moulds. This resulted in the dosages of $24 \mu \mathrm{g}, 48 \mu \mathrm{g}$ and $96 \mu \mathrm{g}$ per carrier (groups 1A, 1B and $1 C$, respectively). Previous experiments show that biologically active rhBMP2 is delivered from these carriers (Schliephake et al., 2015b). The dosage levels of rhBMP2 were chosen based on previous experiments with pure PDLLA carriers showing that in vivo induction of bone tissue occurred at dosages between $400 \mu \mathrm{g}$ of rhBMP2 per $g$ of polymer and the maximum applicable dosage of $1600 \mu \mathrm{g}$ of rhBMP2 per $g$ of polymer (Schliephake et al., 2007; Gruber et al., 2009).

For rapid delivery, blank PDLLA/CaCO 3 carriers were fabricated and loaded with $1.0 \mathrm{~mL}$ solution containing the respective growth factors dosage (24 $\mu \mathrm{g}, 48 \mu \mathrm{g}$ and $96 \mu \mathrm{g}$ per carrier; groups $2 \mathrm{~A}, 2 \mathrm{~B}$ and $2 \mathrm{C}$, respectively). The solution was applied to the surface of the carriers and left for adsorption for $30 \mathrm{~min}$, before implantation.

Blank PDLLA/CaCO ${ }_{3}$ carriers without growth factor loading served as empty control (group 0).

\section{Evaluation of growth factor release from the scaffolds}

Blank scaffolds and scaffolds loaded with the aforementioned amounts of rhBMP2, for both rapid and retarded delivery, were immersed into cell culture medium [Dulbecco's modified Eagle's medium (DMEM) containing $1 \mathrm{~g}$ of glucose; Gibco)] in bovine serum albumin (BSA)-coated 24 well plates (Sigma-Aldrich). The complete medium was collected daily and rhBMP2 release was assessed by pooling the respective aliquots after $24 \mathrm{~h}, 3 \mathrm{~d}$ and every week thereafter until day 35. rhBMP2 content was assessed using a custom-made, commercially produced ELISA sandwich (Dr Mark Hennies, Euskirchen, Germany). The minimum rhBMP2 detectable amount was 100 pg/ $\mathrm{mL}$. Measurements were performed in triplicate for each scaffold. Three scaffolds were evaluated per dosage group. In the groups with adsorbed growth factor and rapid release, after day 7, the evaluation was only carried out every two weeks, as most of the growth factors had already been released after the first week. Release data from the 5 week period were processed by curve fitting, using Excel routines (Microsoft, products.office.com), in order to estimate the amount of growth factor that was released during the scheduled in vivo observation period of 26 weeks. The experimental data recorded for fast and retarded cumulative rhBMP2 release were fitted into a simple exponential function for fast delivery

$$
Q(t)=Q_{\infty}\left(1-e^{-\lambda_{f} t}\right)
$$

and a double exponential function for retarded delivery

$$
Q(t)=Q_{\infty}\left(1-\frac{1}{2} e^{-\lambda_{f} t}-\frac{1}{2} e^{-\lambda_{s} t}\right)
$$

where $Q_{\infty}$ is the extremal value for infinite times and 
$\lambda_{\mathrm{f}}$ and $\lambda_{\mathrm{s}}$ denote the fast and slow time constants of fast surface desorption and slow diffusion through a matrix type delivery system.

\section{Surgical procedures/implantation of carriers}

The ability of the scaffolds to induce bone formation in vivo was assessed by implantation of the carriers into fresh, non-healing defects of the mandibles and into the gluteal muscles of 24 adult male Wistar rats (Rattus norvegicus, average weight $460 \mathrm{~g}$, weight range 310-510 g; Charles River, Sulzfeld, Germany). For implantation into the mandible, the mandibular angle was exposed by performing a submandibular incision and a non-healing defect was created in the mandibular bone, inferior to the mandibular canal (Fig. 1a). In ectopic sites, carriers were implanted into the glutaeus maximus muscle after incision of the skin overlying the muscle. All surgical procedures, housing and animal care were carried out according to the regulation of the State of Lower Saxony, Germany (Regulation No. 33.9-42502-04-10/023). Animals were held and treated in compliance with the ARRIVE guidelines (Web ref. 1). They were held at $20-24{ }^{\circ} \mathrm{C}$ in Makrolon ${ }^{\circledR}$ standard cages $\left(1865 \mathrm{~cm}^{2}\right.$ area), with LIGNOCEL ${ }^{\circledR}$ Select bedding (Rettenmair, Rosenberg, Germany) and a $12 \mathrm{~h}$ light/dark cycle. Animals were fed water and pellets (10 mm) (v1184-0; Ssniff, Soest, Germany) ad libitum. In the experimental set up, they were divided into three dosage groups and one control group. In the mandibles of the control group (group $0, n=6$ ) blank carriers were implanted on one side, whereas the other side was left empty (Fig. 1b). In ectopic sites of the control animals, the blank carriers were implanted only on one side. In the test groups, the retarded delivery carriers and rapid release carriers were implanted one on the right and one on left side. Carriers with the three dosage levels were implanted into the mandibular and ectopic sites of 6 animals each (Fig. 1b). All surgical interventions were performed under general anaesthesia [intraperitoneal (i.p.) injection of $70 \mathrm{mg} / \mathrm{kg}$ ketamine and $0.3 \mathrm{mg} / \mathrm{kg}$ metedomodin] with additional pre- and post-operative analgesia by subcutaneous (s.c.) injection of buprenorphine (up to $0.05 \mathrm{mg} / \mathrm{kg} / \mathrm{d}$ ). Pre- and post-operatively, the status and welfare of the animals were checked daily.

Animals were evaluated after 26 weeks, with the carriers being removed together with the surrounding soft tissue and fixed immediately in $4 \%$ bufferedformalin. Specimens were embedded into Technovit ${ }^{\circledR}$ (Kulzer GmbH, Wehrheim, Germany), according to Donath (1985). For histological analysis, the carriers in the mandibles and the ectopic carriers were cut through the carrier centres. In the mandible, this was done in a plane parallel to the lower border of the mandible. One resulting half of each carrier was used for histology and quantification of bone formation. Three consecutive non-decalcified thick section specimens ( 20-30 $\mu$ m thickness) were produced from the cut surface of each carrier and used for histology. From the other halves of the carriers, six consecutive thin sections $(\sim 5 \mu \mathrm{m})$ were produced from the cut surfaces and used for immunohistochemistry, three of them for alkaline phosphatase (AP) and Runx2 expression, respectively. With six animals per dosage group, each subgroup (rapid/retarded; orthotopic/ ectopic) had 18 specimens studied for histology and 18 specimens studied for both AP and Runx2 expression.

\section{Evaluation of in vivo bone formation}

Assessment of the volume of newly-formed bone was explored using $\mu \mathrm{CT}$ analysis ( $\mu \mathrm{CT}$ device: Skyscan-1272, Bruker), as done in previous reports (Ueyama et al., 2016; Rakhmatia et al., 2017). Unfortunately, this approach used at the mandible site showed that discrimination based on density values did not allow for distinguishing between the newly-formed and the pristine bone after 6 months of healing, presumably due to the long healing

a
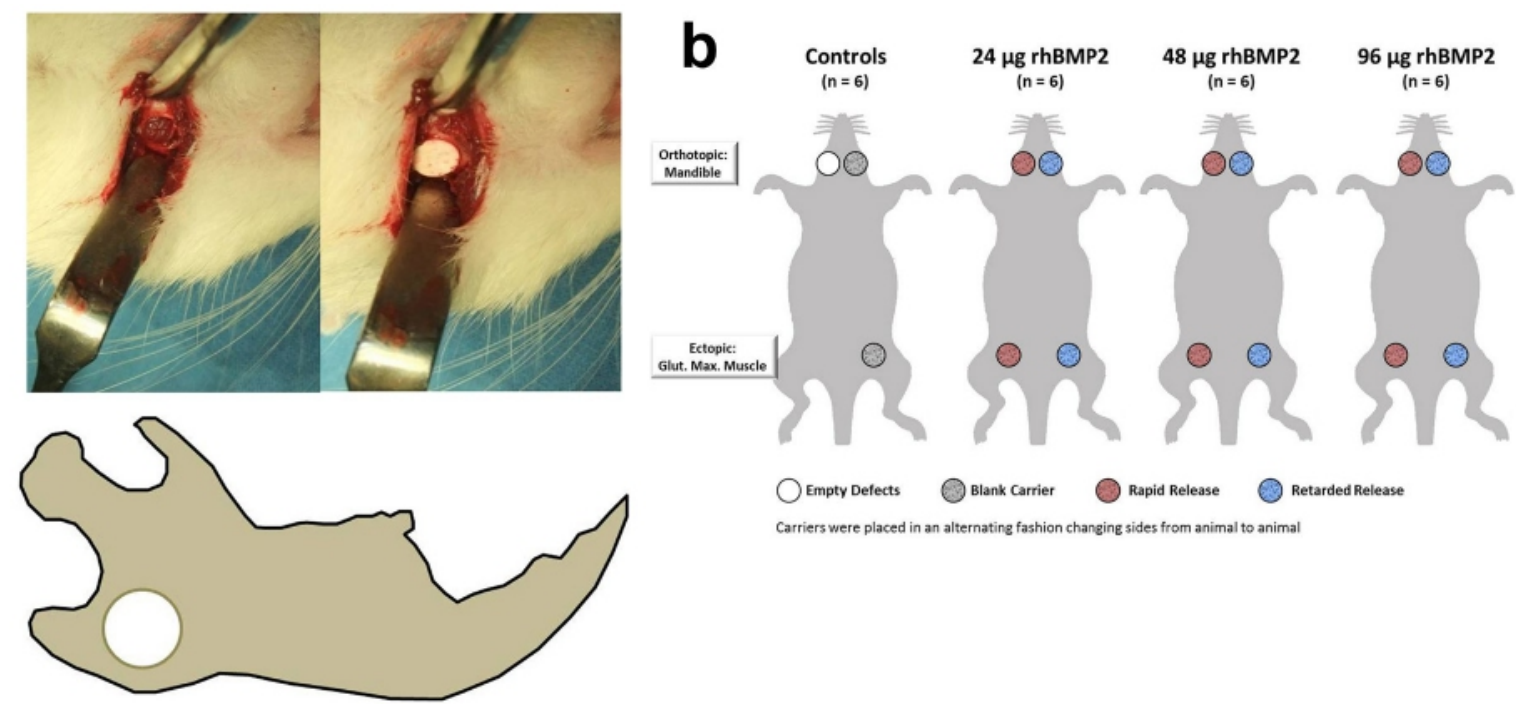

Fig. 1. (a) Above left: creation of a circular mandibular defect; above right: press fit insertion of the carrier; below: schematic representation of a rat mandible with location of the mandibular defect in the mandibular angle underneath the mandibular canal. (b) Schematic representation of the study design. 

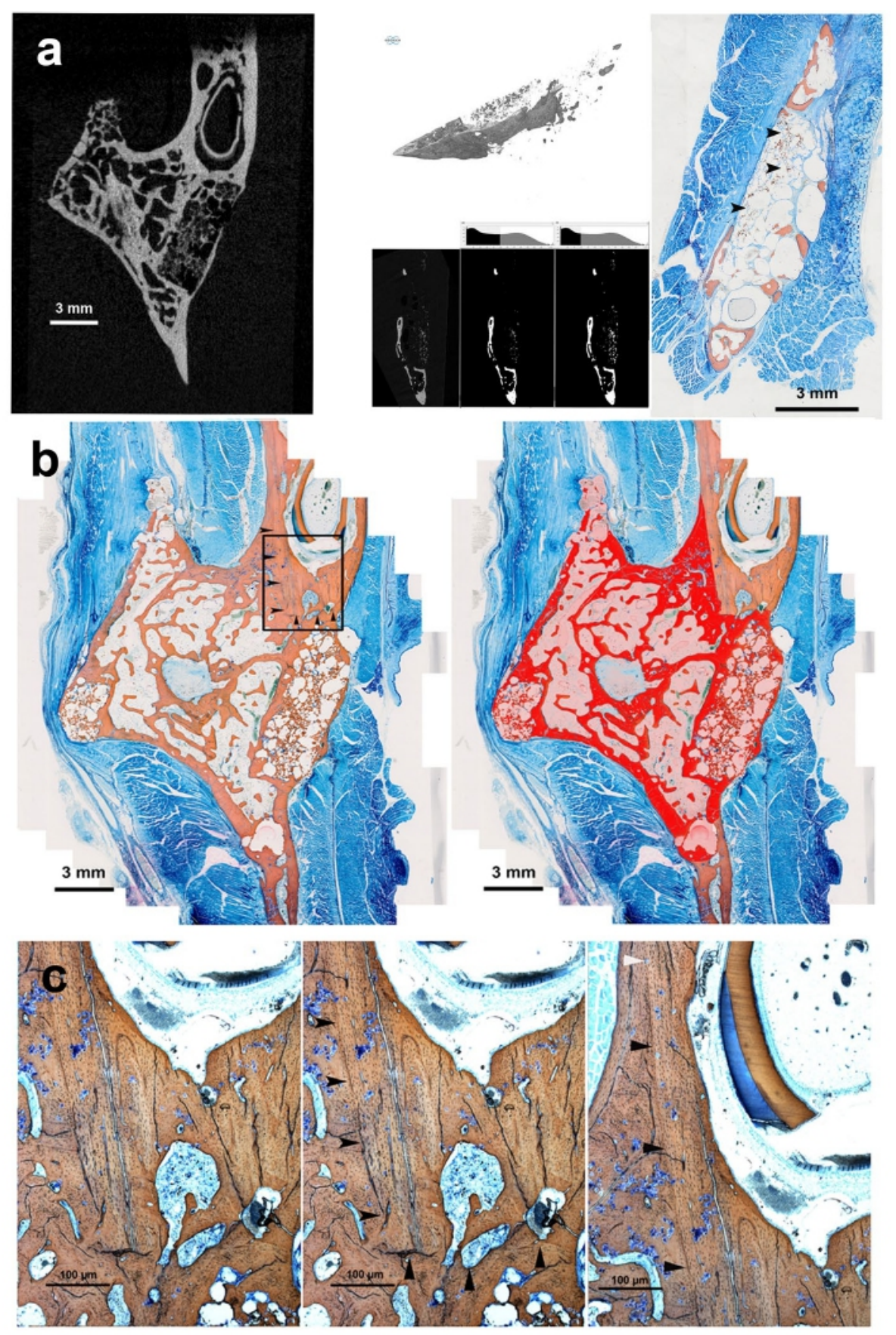

Fig. 2. (a) Left: $\mu \mathrm{CT}$ showing a cross section through a mandibular defect with the cross sectioned carrier. Note that regenerated bone lateral of the mandible was not clearly distinguishable from pristine bone (source voltage $60 \mathrm{kV}$, source current $166 \mu \mathrm{A}$, image pixel size $16.78 \mu \mathrm{m}$, exposure $1331 \mathrm{~ms}$, flatfield correction, filter $0.25 \mathrm{~mm} \mathrm{Al}$ ). Right: compilation of $\mu \mathrm{CT}$ data and a corresponding histological specimen of an ectopic carrier. $\mu \mathrm{CT}$ evaluation: upper part, $3 \mathrm{D}$ rendering of $\mu \mathrm{CT}$ data, indicating that the display of newly-formed bone was blurred by $\mathrm{CaCO}_{3}$ particles in the scaffold parts not occupied by bone tissue; lower part, three versions of a cross-sectional plain: left image includes all density values, right image displays binary imaging with wide density threshold (60-255) and central image displays binary image using a narrower range of threshold values with the lower threshold value chosen to best represent clearly identifiable bone tissue. Threshold values are indicated above the 2D images. Arrow heads in the micrograph indicate location of $\mathrm{CaCO}_{3}$. (b) Alizarin red-methylene blue staining. Left: cross section through a mandibular carrier. Arrow heads indicate line between newly-formed bone and pristine bone; frame represents area displayed in (c). Right: manually isolated region of interest (light red) with digitised area (pixels) of newly-formed bone in full red. (c) Alizarin red-methylene blue staining. Left: higher magnification of the transition zone between old and newly-formed bone. Structure and pattern of osteocytes allowed for discrimination between pristine and newly-formed bone (see frame in $\mathbf{b}$ ). Centre: black arrow heads marking the line between old and new bone. Right: further anterior, the line between old and new bone merged with periosteal appositional bone formation (white arrow head). 
period of 26 weeks (Fig. 2a). During evaluation of ectopic carriers, we realised that carrier portions not penetrated by newly-formed bone exhibited mineral dense $\mathrm{CaCO}_{3}$ particles that impaired precise identification of bone tissue, even applying different density thresholds (Fig. 2b). Therefore, non-decalcified thick sections were produced from the scaffolds, according to Donath (1985), and the specimens surface stained with alizarin redmethylene blue staining. Newly-formed bone area was evaluated by histomorphometrical analysis of cross sections through the centre of each carrier. For histomorphometry, the specimens were mounted on a scanning table (Olympus dotSlide ${ }^{\circledR}$; Olympus Life Science, Hamburg, Germany) and the micrographs were serially recorded using a Sony 3CCD video camera (Germany) at $100 \times$ magnification, digitised and mounted together into one picture. Three slides per carrier were evaluated. Each histological slide was scanned at a magnification of $100 \times$ into one large file. For quantification of bone formation, the whole area of newly-formed bone per slide was assessed using a stereological representation of newly-formed bone volume. Newly-regenerated bone tissue was identified in the histological cross sections and manually isolated from pristine bone by outlining the respective area. Bone tissue in this area was identified by filters adapted for the red colour of the stained bone tissue (Adobe Photoshop CS5; Adobe Systems $\mathrm{GmbH}$, Munich, Germany) (Fig. 2c). Areas with unclear discrimination between pristine and newlyformed bone were evaluated at higher magnification for manual selection (Fig. 2d). The area occupied by newly-formed bone was quantified by the pixel counting routines of the software. Mean values per carrier were calculated from the individual slides and average values for each group were calculated from the mean values of the respective carriers.
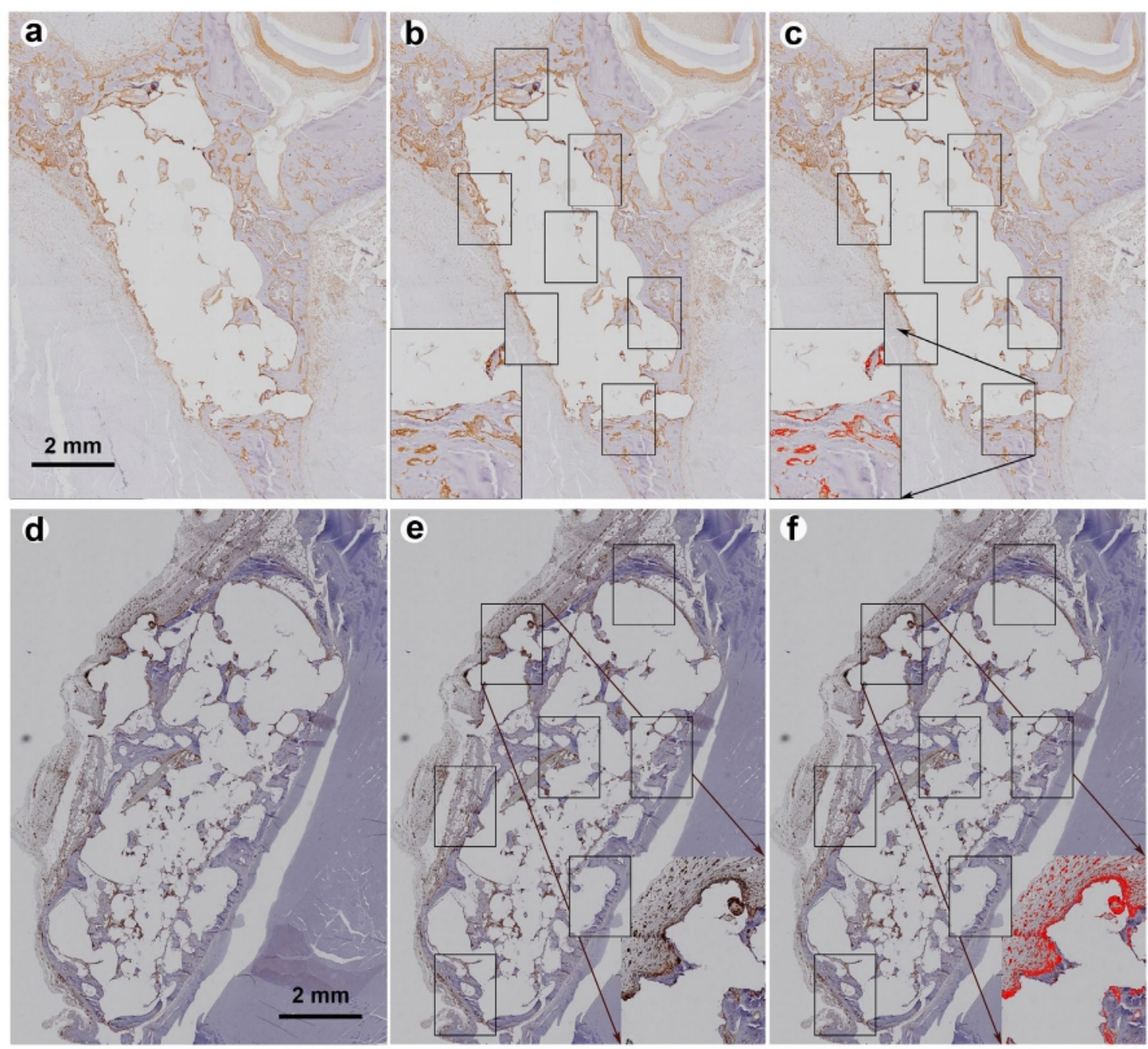

Fig. 3. (a) Micrograph of a mandibular carrier with immunohistochemistry staining for AP. (b) Location of the 7 regions of interest across the cross section of the carrier with one region of interest at higher magnification with positive staining for AP. (c) Illustration of digitised area (pixels) positive for AP; bar indicates $2 \mathrm{~mm}$. (d) Micrograph of an ectopic carrier with immunohistochemistry staining for Runx2. (e) Location of the 7 regions of interest across the cross section of the carrier with one region of interest at higher magnification with positive staining for Runx2. (f) Illustration of digitised area (pixels) positive for Runx2; bar indicates $2 \mathrm{~mm}$. 


\section{Evaluation of in vivo induction of bone specific markers}

Induction of bone specific markers was assessed using immunohistochemistry. AP was chosen as a marker involved in mineralisation and Runx2 (Cbfa1) was selected as a transcription factor involved in osteogenic differentiation. $5 \mu \mathrm{m}$-thin, non-decalcified sections were produced from the centres of the carriers and analysed by immunohistochemical staining. No decalcification was performed in order to preserve, as much as possible, the respective epitopes. Antigen demasking was performed using EDTA/Tween 20 (pH 8.0) for $18 \mathrm{~h}$ at $60^{\circ} \mathrm{C}$, followed by AP staining and Target Retrieval buffer treatment ( $\mathrm{pH}$ 6.1; S1699; DAKO). Blocking of endogenous peroxidase was performed using DAKO peroxidase blocking reagent S2023. Endogenous rat IgG were blocked using $200 \mu \mathrm{L}$ of Rodent Block R (RBR62; Biocare) for $60 \mathrm{~min}$. Isotype controls were performed using $15 \mathrm{mg} / \mathrm{mL}$ of negative control rabbit IgG (X036; DAKO). For AP staining, $0.5 \mu \mathrm{g} / \mathrm{mL}$ of polyclonal rabbit anti-bone alkaline phosphatase (ab108337; Abcam) in conjunction with HRP-polymer kit Biocare (RMR622)/XR factor XRF 964 were used. For Runx2, $1 \mu \mathrm{g} / \mathrm{mL}$ of polyclonal rabbit anti-Runx2 (ab23981; Abcam) with $60 \mu \mathrm{L}$ of secondary swine-anti rabbit antibody (1 : 100 dilution) (P0217; DAKO) were used. DAKO chromogen system K3468 was used for staining.
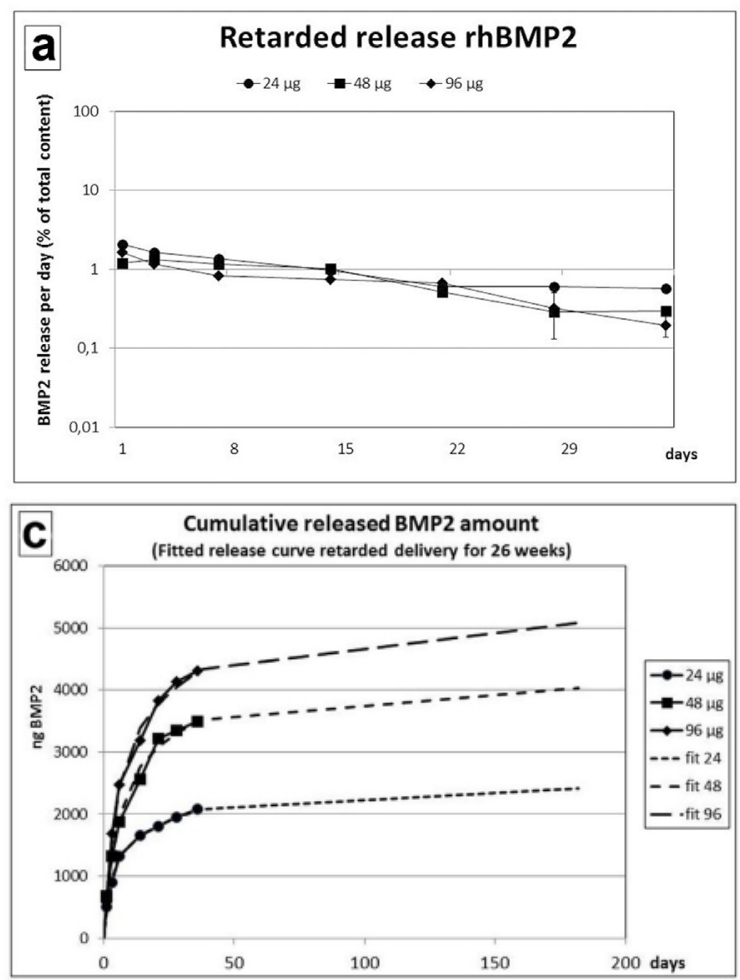

For evaluation of positively-stained areas, each stained slide was scanned at magnification of $100 \times$ and combined into one image, as described. At that magnification, each pixel had a width of $\sim 1.3 \mu \mathrm{m}$. The magnification required for the identification of positively stained areas precluded the evaluation of the complete cross section of the regenerated bone/ carrier. For quantification, seven frames of $670 \times 500$ pixels, corresponding to $864 \times 661 \mu \mathrm{m}$ each, were placed over the area of regenerated bone in contact with the carrier material, both in the mandible and the gluteus maximus. Six of these frames were placed in the periphery of the regenerated bone area and one in the centre (Fig. 3a-f). Every effort was made to place the frames at even distances to each other. In empty mandibular defects, the frames were positioned in a linear fashion at even distances between the two edges of the defect.

Induction of AP and Runx2 was quantified applying adapted colour filters (Adobe Photoshop CS5, Adobe Systems GmbH, Munich, Germany), for the identification of positively stained cells, and expressed by the counted number of positively stained pixels. Average values per field were calculated for each specimen (slide). From these, mean values for each carrier were calculated and finally group mean values were calculated from the average values of the individual carriers.
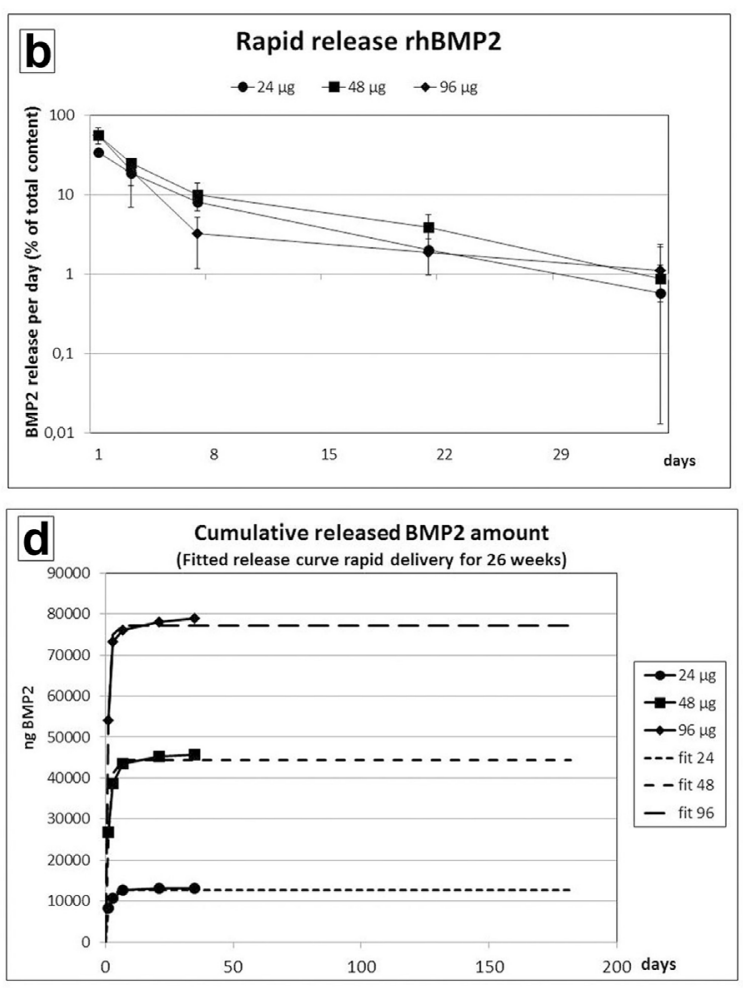

Fig. 4. (a) Release of BMP2 from retarded release carriers $(n=3)$; symbols represent mean values, error bars represent standard deviation, log scale y axis. (b) Release from rapid release carriers $(n=3)$; symbols represent mean values, error bars represent standard deviation, log scale y axis. (c) Release characteristics of retarded release carriers. Curve fitting suggested that in the period of 5 to 26 weeks between 400 and 700 ng of BMP2 were likely to be additionally released. (d) Release characteristics of rapid release carriers. Curve fitting showed that no further release could be expected after 5 weeks. 


\section{Statistics}

Analyses of variance (ANOVA), as well as, paired t-tests (IBM SPSS Statistics 24.0, http://support.spss. com) were used to compare the induction of AP and Runx2 in vivo and the amount of bone formed in vivo, around and inside the scaffolds. Bonferroni correction was used in case of multiple testing. The significance level was set to $p<0.05$.

\section{Results}

\section{Release of growth factors in vitro}

The retarded release carriers delivered between $1.2 \%$ and $2.1 \%$ of the incorporated rhBMP2 on day 1 , with a decrease over the following 5 weeks to levels of 0.2 $0.5 \%$ per interval. The rapid release carriers exhibited a burst release between 34.2 and $56.2 \%$ of the loaded growth factors on day 1 , with a decrease to $0.5-1.1 \%$ after 5 weeks (Fig. 4a,b). The curve fitting resulted in an estimated delivery from the retarded release carriers of 2.40-5.08 $\mu \mathrm{g}$ of rhBMP2 after 26 weeks, whereas the delivery from the rapid release carriers was calculated not to increase above that amount delivered after 5 weeks - varying between 12.69 and $79.01 \mu \mathrm{g}$ of rhBMP2, according to the initial dosage applied (Fig. 4c,d).

\section{In vivo bone formation}

No adverse reactions were observed and uneventful healing occurred, so that all animals were available for evaluation.

In mandibular sites, there was no obvious difference in morphology between the bone induced by retarded $v s$. rapid delivery of rhBMP2 (Fig. 5a-f). Increasing growth factor dosages led to increasingly dense bone formation. Interestingly, the carrier exhibited a more advanced degree of resorption in the case of more bone formation, due to a larger dosage of rhBMP2. In ectopic sites, bone induced by retarded delivery carriers was more evenly distributed across the carrier cross section than in rapid release carriers, where bone formation in the low and medium dosage groups occurred in smaller amounts, mainly at the
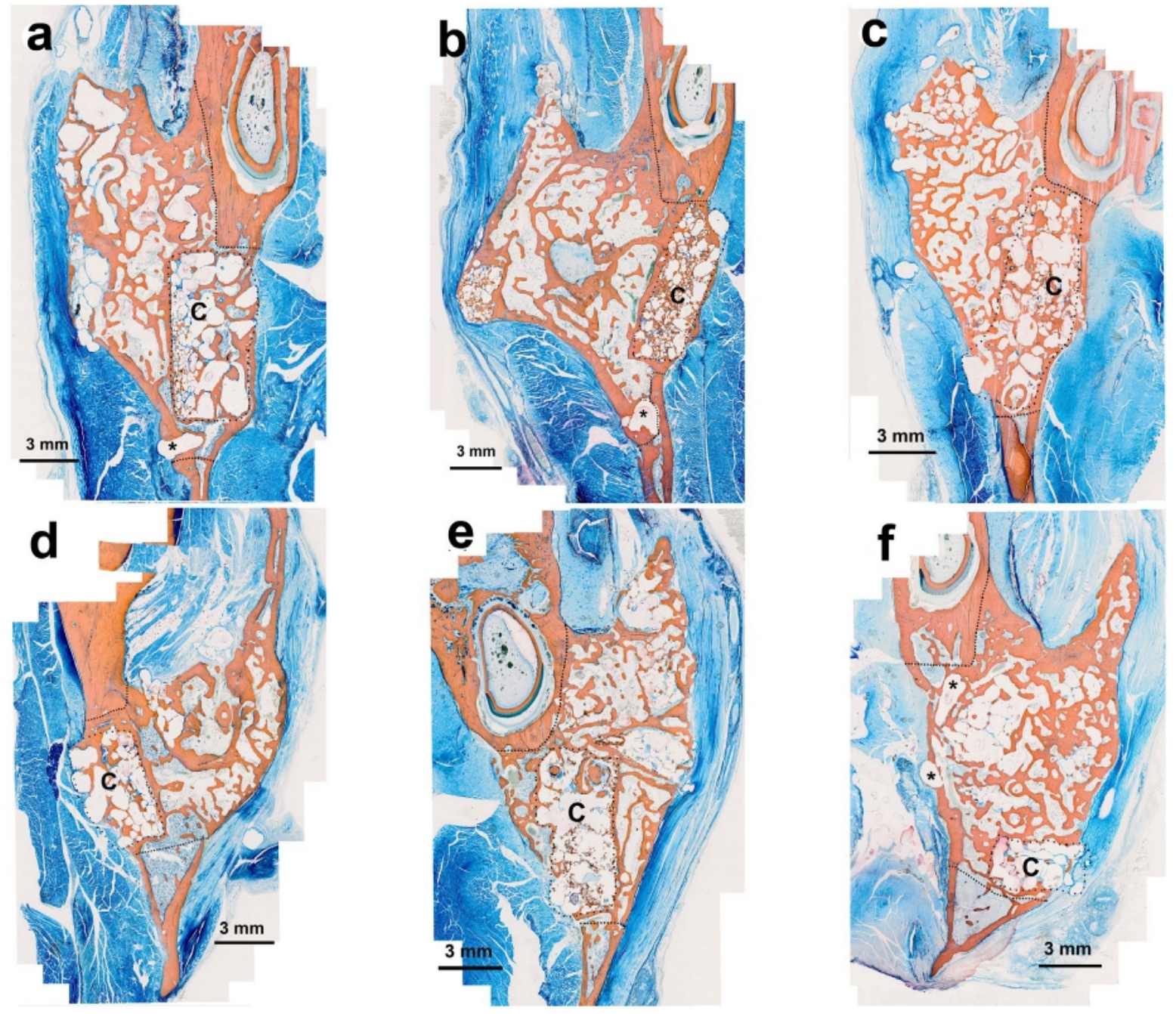

Fig. 5. (a-c) Alizarin red-methylene blue staining showing bone formation at the mandible site after retarded release of (a) $24 \mu \mathrm{g}$, (b) $48 \mu \mathrm{g}$ and (c) $96 \mu \mathrm{g}$ of rhBMP2. (d-f) Alizarin red-methylene blue staining showing bone formation at the mandible site after rapid release of (d) $24 \mu \mathrm{g}$, (e) $48 \mu \mathrm{g}$ and (f) $96 \mu \mathrm{g}$ of rhBMP2. Densely dotted line marks the border between pristine and newly-formed bone; loosely dotted line marks the remnant of the carriers, ${ }^{*}$ identifies dislocated carrier particles. 
periphery of the carrier. Only in the high dosage group, was bone formation also seen penetrating the rapid delivery carriers (Fig. 6a-f).

Empty mandibular defects failed to heal within the observation period. Empty carriers in mandibular defects exhibited only minimal bone ingrowth. Empty carriers in ectopic sites exhibited no bone regeneration (Fig. $7 \mathrm{a}-\mathrm{c}$ ).

Histomorphometrical evaluation of bone formation in mandibular sites revealed a dosedependent increase in bone formation in the groups with retarded release of $\mathrm{rhBMP} 2$. There were significant differences between empty defects/blank scaffolds and all BMP-groups, as well as, between the high dose and the other two dosage groups (Fig. 8a). Groups with rapid release exhibited significantly increased bone formation over empty defects/blank controls, with significantly smaller areas of bone formation in the low dose carriers compared to the other two dosage groups. There was no significant difference in the quantity of bone formation between rapid and retarded delivery in any of the dosage groups.

In ectopic sites, blank carriers did not induce any bone formation. Both rapid and retarded release carriers induced a dose-related increase in bone formation (Fig. 8b). In general, the area of ectopic bone formation ranged between 0.2 and $10.4 \%$ of the area of mandibular bone formation, depending on the applied dosage. This was significantly lower compared to the extent of mandibular bone formation in all dosage and delivery groups. In groups with retarded release of rhBMP2, significant differences were found between low dose carriers and the other two dosage groups. In groups with rapid delivery of rhBMP2, significant differences were seen only between the low and the high dosage group. Comparing bone formation between retarded delivery and rapid release groups, the retarded release carriers induced significantly more bone
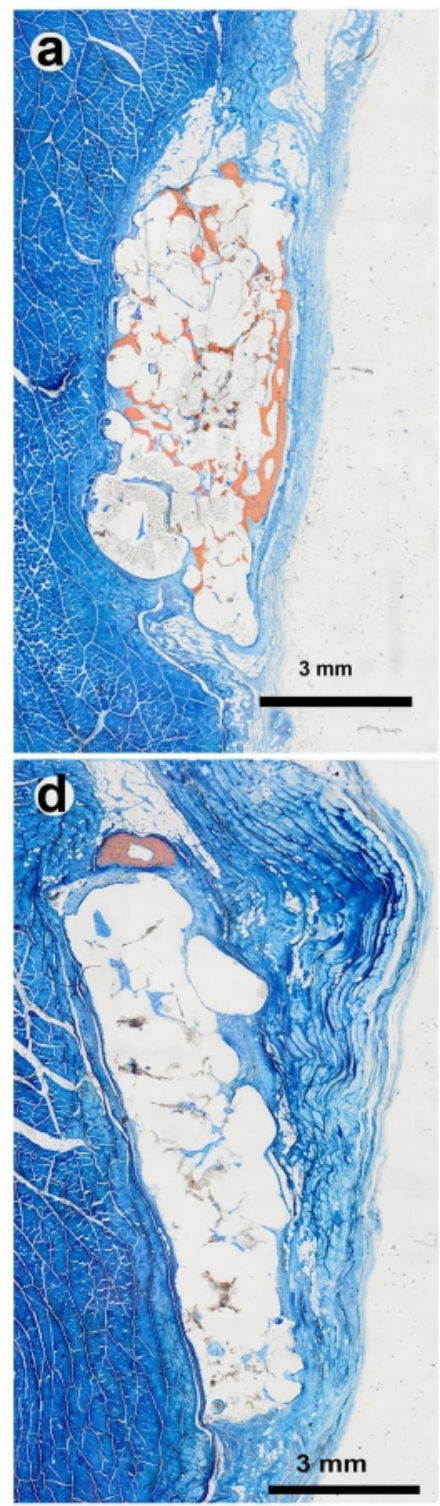
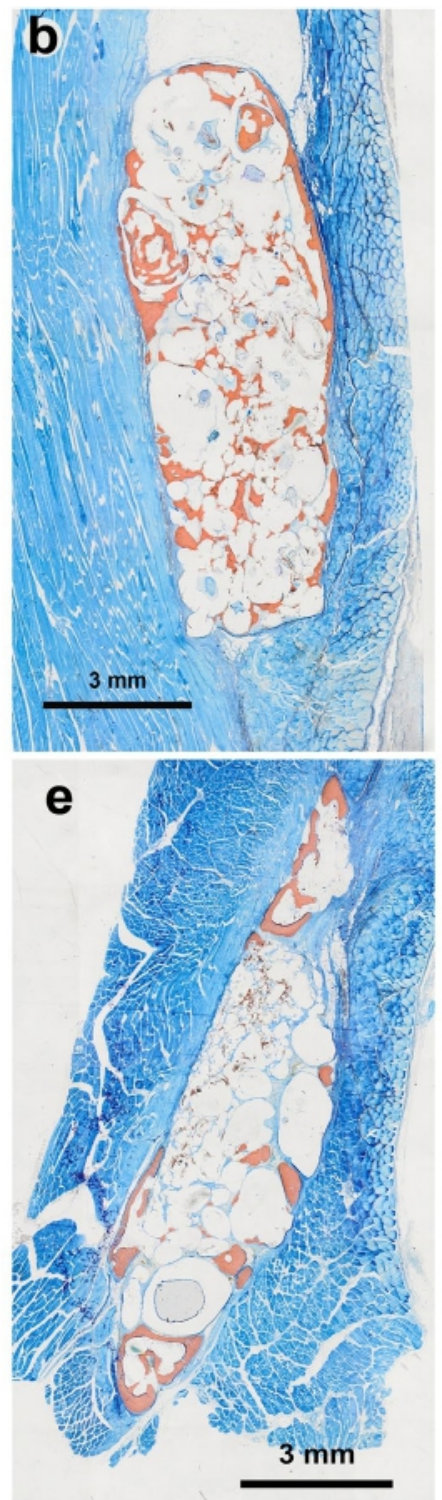
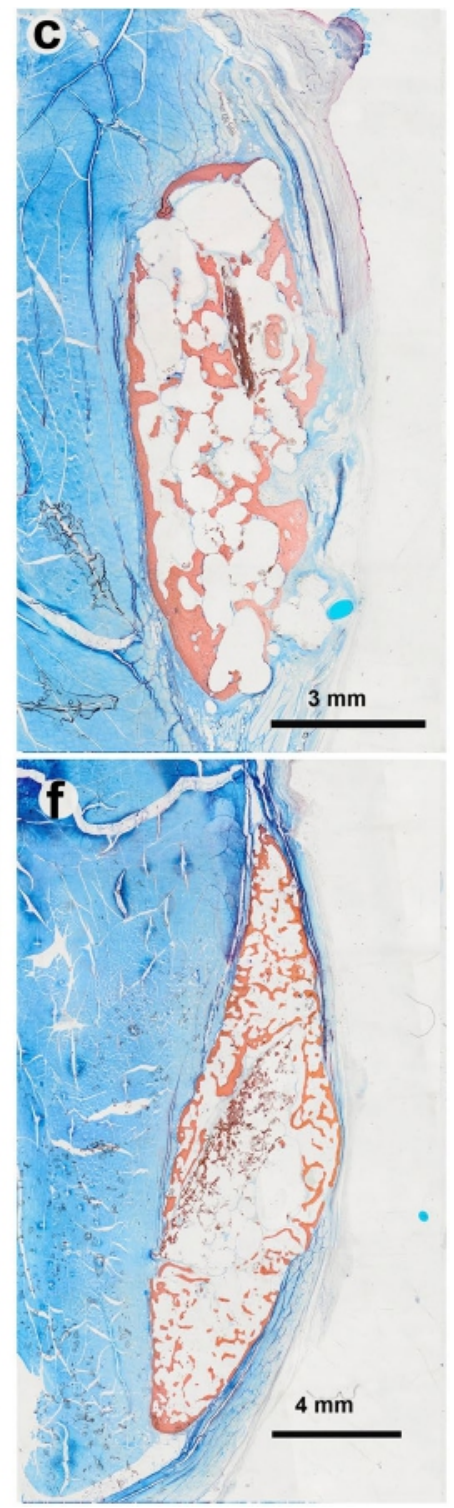

Fig. 6. Bone formation at the ectopic site after retarded release of (a) $24 \mu \mathrm{g}$, (b) $48 \mu \mathrm{g}$ and (c) $96 \mu \mathrm{g}$ of rhBMP2 and rapid release of (d) $24 \mu \mathrm{g}$, (e) $48 \mu \mathrm{g}$ and (f) $96 \mu \mathrm{g}$ of rhBMP2. (a-f) Alizarin red-methylene blue staining. 
formation than the rapid delivery carriers in the low and medium dosage groups.

\section{In vivo induction of bone specific markers Alkaline phosphatase}

In ectopic sites, induction of AP by rapid release carriers was visible in small nests of cells in soft tissue, growing into the pores of the carriers, as well as, in cells lining the newly-formed bone (Fig. 10a-c). In the mandible, rapid delivery carriers exhibited a less intense staining. Cells were more scattered in fewer locations and less densely accumulated both in the soft tissue and in the vicinity of the newly-formed bone (Fig. 10d-f). In carriers with retarded delivery, similar features were seen. Similarly to rapid delivery carriers, the number of positively stained nests of cells and the density of positive cells in these areas was higher in ectopic carriers when compared to mandibular carriers (Fig. 10g-i,k-m).

Quantitative evaluation of mandibular sites exhibited a dose-dependent increase in AP expression, in and around both retarded and rapid release carriers, that was significantly higher than in empty defects and around blank controls - except for the low dosage carriers with rapid release. Low dose carriers with retarded delivery exhibited a significantly higher AP expression compared to low dose carriers with rapid release (Fig. 11a). Ectopic sites showed a dose-dependent increase of AP compared to blank controls, in carriers with both rapid and retarded delivery of rhBMP2 (Fig. 11b). Interestingly, the area of positive staining in ectopic sites was between 8.3 and 16.5 times larger than in mandibular sites. These differences were significant for all the three dosage groups and both modes of delivery. Retarded release carriers induced significantly higher levels of expression of AP in the medium and high dosage groups compared to blank carriers, whereas rapid release carriers induced significantly increased AP expression only in the high dosage group. There were significant correlations between the area of bone formation and the expression of AP in mandibular sites, for both rapid and retarded release carriers $(r=0.592, p=0.006$ and $r=0.557, p=0.013$, respectively). Ectopic sites exhibited a significant correlation between bone formation and expression of AP only in groups with retarded release carriers $(r=0.605, p=0.002)$, whereas no correlation was found with rapid release carriers $(r=0.366, p=0.135)$.

\section{Induction of Runx2}

In mandibular sites, expression of Runx 2 was detected next to newly-formed bone, but also distributed across the non-osseous tissues, inside the carriers with both rapid and retarded delivery of rhBMP2 (Fig. 12a-c,g-i). In ectopic sites, expression was lower in both delivery groups, with scattered islands of positive cells in non-osseous tissue in the low dosage groups (Fig. 12d,k). In the high dosage groups, more intense staining was observed adjacent to newly-forming bone tissue, for both rapid and retarded release carriers (Fig. 12e,f,l,m).

Quantitative assessment revealed that expression of Runx2 in mandibular sites was significantly increased compared to empty defects/blank carriers in all groups, with both retarded and rapid release of rhBMP2 (Fig. 13a). In general, the level of expression was comparable to that of AP. For the retarded delivery of rhBMP2, there was no significant dosedependent increase observed between the medium and the high dosage group. The mode of delivery had a significant effect on Runx2 expression level in the high dosage group $(p=0.031)$, with a near significant difference also in the medium dosage carriers $(p=0.069)$. In all dosage groups, ectopic sites exhibited a significant increase in Runx2 expression for both retarded and rapid release carriers, compared to blank carriers. A significant difference in the expression of Runx2 was only found between the low and high dosage group in rapid delivery

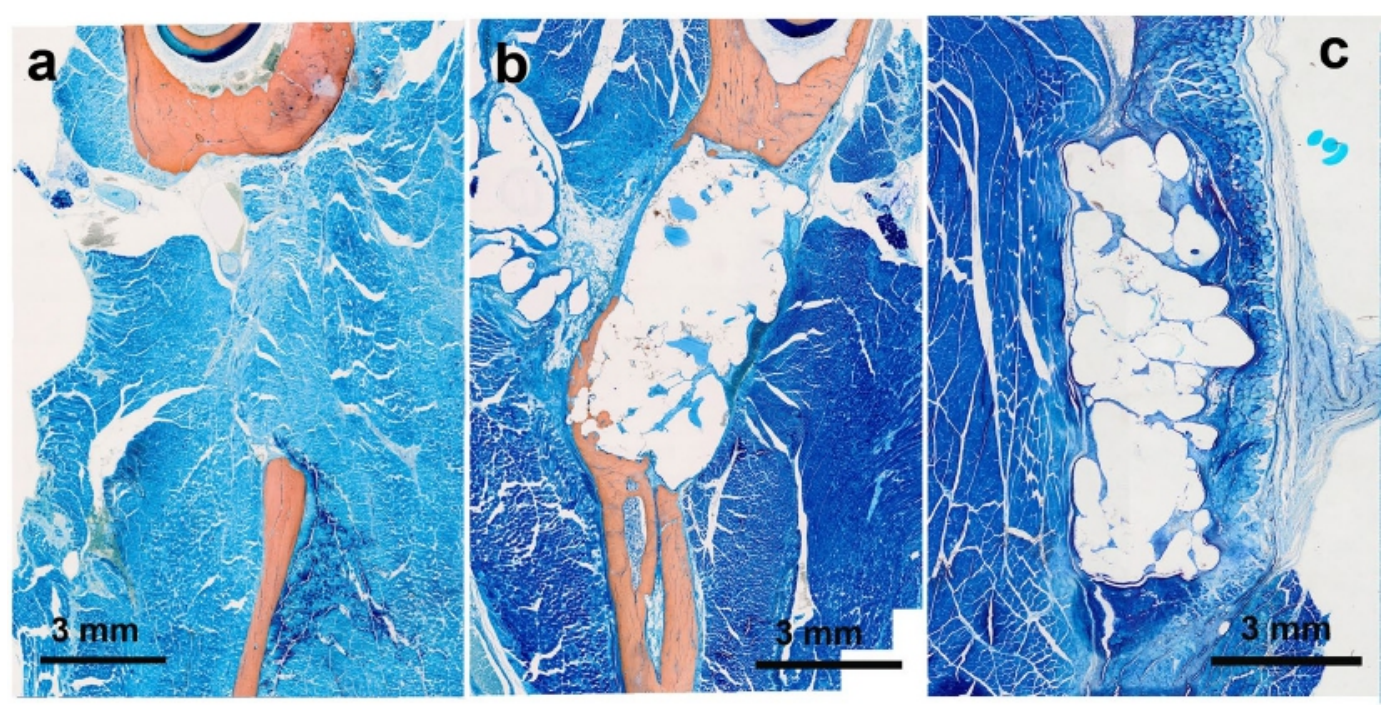

Fig. 7. (a) Empty mandibular defect showing failure to heal within the observation period of 26 weeks. (b) Mandibular defect filled with blank carrier with minimal bone regeneration at the edges. (c) Ectopic blank carrier devoid of bone regeneration. (a-c) Alizarin red-methylene blue staining. 
carriers (Fig. 13b). No significant differences were found between rapid and retarded release in the three dosage groups. The area of positive staining ranged between 23.0 and $91.3 \%$ compared to the mandibular site, with significantly lower expression in ectopic than in mandibular sites in all but one dosage group. In mandibular sites, a significant correlation between the expression of Runx 2 and the degree of bone formation was found for both rapid and retarded delivery of rhBMP2 $(r=0.604$, $p=0.004 ; r=0.881, p<0.001)$. In ectopic sites, only the retarded release exhibited a correlation between bone formation and expression of Runx2 $(r=0.526$, $p=0.008)$, whereas rapid delivery did not show such correlation $(r=0.366, p=0.135)$.

Control specimens, from both mandibular and ectopic sites, did not show positive staining for neither AP nor Runx2 (Fig. 14a-f).

\section{Discussion}

There is widespread consensus that bone growth factor delivery for tissue regeneration purposes should be accomplished through retarded release, although exact dosages and quantitative patterns of delivery for the regeneration of bone tissue are largely unknown (Gothard et al., 2014). Among the many studies on successful retarded delivery of BMPs, there are reports that mention a lack of effect of retarded release with respect to the amount of bone formed (Geuze et al., 2012; Gruber et al., 2015). The present study suggested that both retarded release, after incorporation of rhBMP2 into a polymer carrier, and rapid release, after adsorption of rhBMP2 onto the carrier surface, could induce a comparable amount of bone formation in mandibular sites, even at lower dosages. Although the calculated dosage, delivered through retarded release, was considerably lower than the rapidly delivered dosage, a larger bone volume would be expected as a result of 26 weeks of retarded delivery than from a few days of rapid release. One of the reasons for the observed lack of quantitative effect of the retarded delivery may be that BMPs are pleoiotropic factors that organise a regenerative environment beyond the sole induction of osteogenic differentiation (Schultz et al., 2014, Kopf et al., 2014, Chalazonitis and Kessler, 2012). For example, it is well known that rhBMP2 alone can promote angiogenesis through the expression of the vascular endothelial growth factor (VEGF) in both osteoblasts and stromal cells (Yang et al., 2015; Deckers et al., 2002). In surgical sites that provide plenty of recruitable cells, such as fresh bone defects in rat mandibles, even a single inductive impulse during a short period of low dose BMP delivery may accomplish a self-sustained regenerative effect through induction of a "regeneratome", in which cellular interactions, required for successful bone formation, continue without further external supply of BMP2. The results from the ectopic sites suggested that the threshold dosage for such an effect depended on the site of growth factor delivery. In ectopic sites, only the high dosage of rapidly delivered BMP2 was able to induce comparable amounts of bone, whereas low and medium dosage levels of BMP2 led to reduced bone formation, when compared to retarded delivery. Thus, the in vivo results confirmed previous in vitro findings, according to which musclederived stem cells require prolonged exposure to BMPs to undergo osteogenic differentiation ( $\mathrm{Li}$ et al., 2013). Therefore, it was possible to hypothesise that, in ectopic sites, a more sustained signal was required to persistently organise the environment for bone regeneration. Moreover, the observation that the amount of ectopic bone formed was significantly lower in all groups compared to the same dosage applied in mandibular sites indicated that, in mandibular sites, the number of recruitable cells was larger and the regenerative environment more inducible in the direction of bone formation. This may have been due to the existence of pristine osteogenic cells in both bone and overlying periosteum.

The immunohistochemical evaluation of markers of osteogenic differentiation additionally suggested that the type of cells induced and/or the signalling
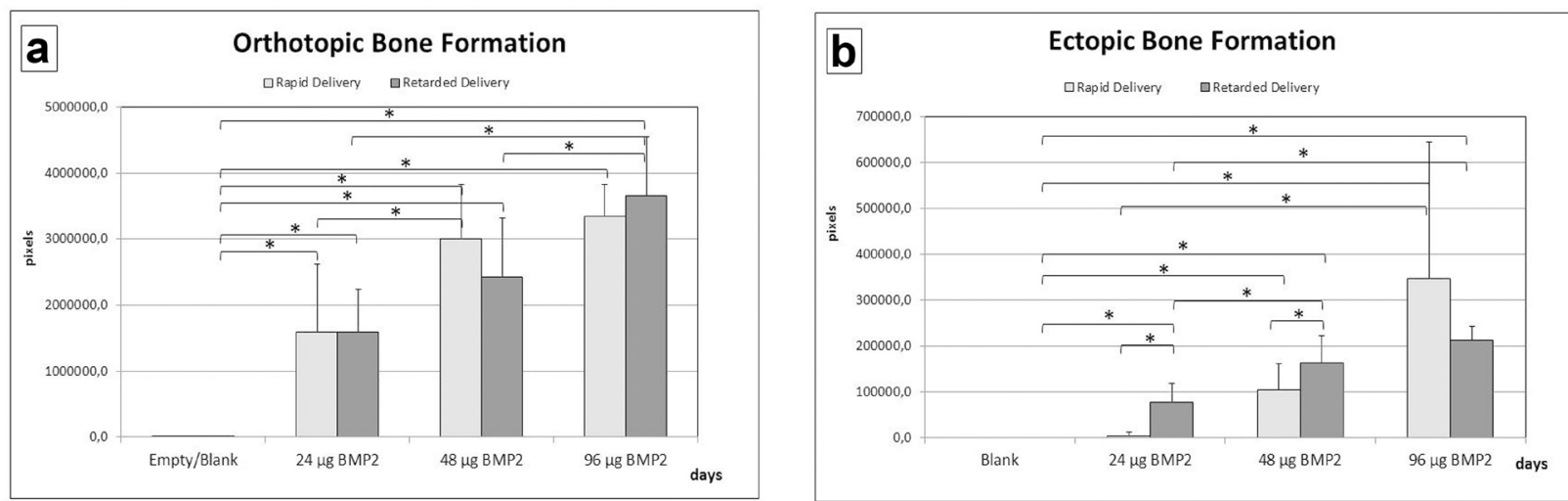

Fig. 8. Quantitative evaluation of bone formation in (a) mandibular sites (b) and ectopic sites. Comparison of paired data subsets (i.e. comparison between rapid and retarded release) was performed by paired $t$-tests. Comparison of unpaired data subsets (comparison of different dosages) was performed by ANOVA with Bonferroni correction; $n=6$, bars represent mean value, error bars represent standard deviation, ${ }^{*} p<0.05$. 
pathway activated may have been different in mandibular vs. ectopic sites. A surprising finding was that AP expression level was significantly higher in ectopic sites compared to mandibular defects in all dosage groups, regardless of the mode of delivery. Additionally, Runx2 expression level was almost consistently lower in both retarded and rapid delivery carriers in ectopic sites compared to mandibular sites. Induction of osteogenic differentiation is reported to be different, with regard to expression of Runx2, depending on the intracellular pathway that is activated upon binding of osteogenic growth factors. Activation of the canonical pathway by BMPs through ERK1/2/Smads1/5/8 phosphorylation involves the expression of osterix through Runx2. On the contrary, activation of the p38/MAP kinase is reported to result in the expression of osterix through Dlx5 or Msx2, without necessarily involving Runx2 expression (Li et al., 2015; Lee et al., 2003, Matsubara et al., 2008). Moreover, mesenchymal progenitor and stromal cells of different origin behave differently upon osteogenic induction and provide different osteogenic characteristics (Sacchetti et al., 2016; Jackson et al., 2009). In conjunction with the quantitative results of bone formation, it was possible to hypothesise that the difference in levels of expression of Runx 2 and AP in ectopic sites compared to mandibular sites reflected the activation of different pathways in different target cell populations.

The levels of expressions of AP and Runx2 in relation to bone formation might be considered as quantitative indicators of a well-orchestrated process of osteogenesis and remodelling. In this respect, it was interesting to note that, in ectopic sites, there was no significant correlation between the levels of expression of both AP and Runx2 and the extent of bone formation in carriers with rapid delivery. In contrast, bone formation after retarded delivery in ectopic sites and mandibular sites, as well as, rapid delivery in mandibular sites showed this correlation. This might indicate that the process of osteogenesis was less uniform in ectopic sites, with rapid growth factor delivery leading to a less effective bone formation at lower and medium dosage levels.
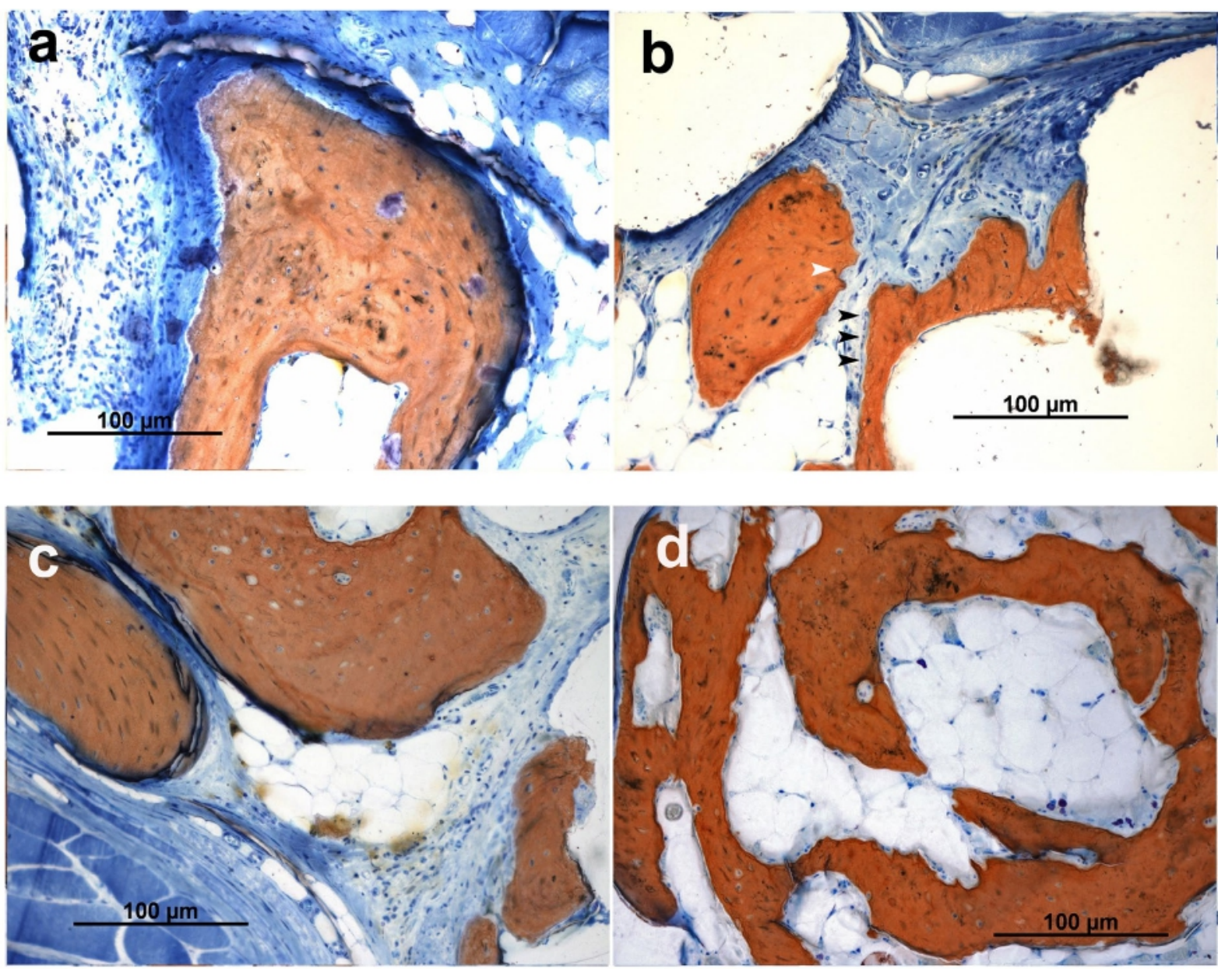

Fig. 9. (a) Ectopic bone formed in a carrier with rapid release of $24 \mu \mathrm{g}$ rhBMP2: inactive rounded contour devoid of active bone remodelling. (b) Ectopic bone formation in a carrier with retarded release of $24 \mu \mathrm{g}$ rhBMP2: remodelling activity indicated by resorption lacunae (white arrow head) and a thin line of osteoid and flat osteoblasts (black arrow heads) (c) Ectopic bone formed in a carrier with rapid release of 48 mg rhBMP2: rounded contour of bone trabeculae without active bone remodelling. (d) Ectopic bone formation in a carrier with retarded release of $48 \mu \mathrm{g}$ rhBMP2: some remodelling activity could be seen with resorption lacunae (white arrow head). (a-d) Alizarin red-methylene blue staining. 


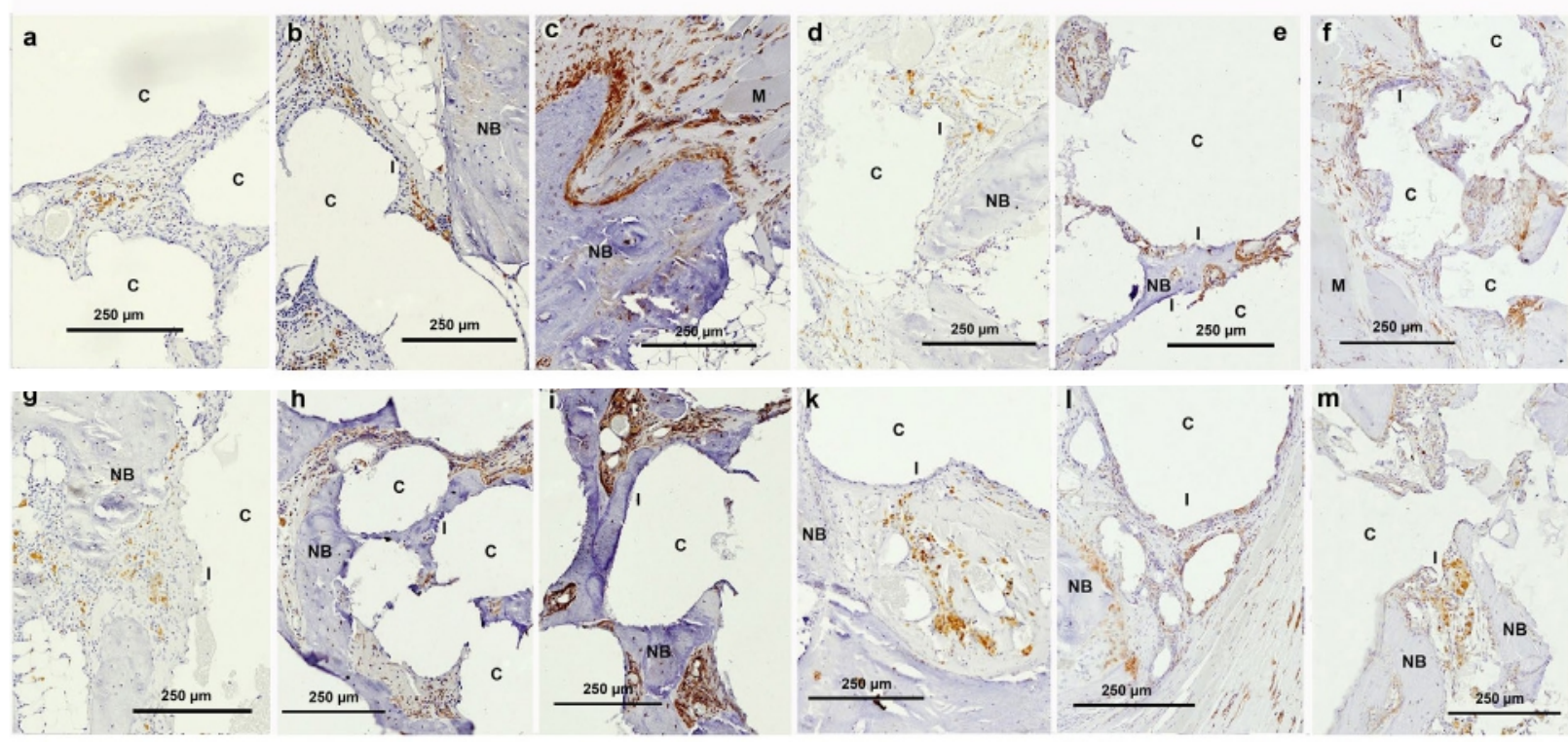

Fig. 10. Representative images of immunohistochemical features of AP staining: (C) carrier material solubilised during histologic processing, (NB) newly-formed bone, (I) interface between carrier surface and newly-formed tissue and (M) muscle tissue are indicated. Rapid delivery of (a) $24 \mu \mathrm{g}$, (b) $48 \mu \mathrm{g}$ (c) and $96 \mu \mathrm{g}$ of rhBMP2 in ectopic carriers: $(\mathbf{a}, \mathbf{b})$ positive staining showed in cell islands, in non-osseous tissue invading the carriers and (c) in cells lining newly-formed bone. Rapid delivery of (d) $24 \mu \mathrm{g}$, (e) $48 \mu \mathrm{g}$ and (f) $96 \mu \mathrm{g}$ of rhBMP2 in mandibular carriers: lower expression of AP in mandibular sites with scattered cells in non-osseous tissue and less densely accumulated cells in the vicinity of newly-formed bone. Retarded delivery of (g) $24 \mu \mathrm{g}$, (h) $48 \mu \mathrm{g}$ and (i) $96 \mu \mathrm{g}$ of rhBMP2 in ectopic carriers: positive staining for AP, seen in cells adjacent to newly-formed bone tissue, increased with increasing dosage of rhBMP2. Retarded delivery of (k) $24 \mu \mathrm{g}$, (l) $48 \mu \mathrm{g}$ and (m) $96 \mu \mathrm{g}$ of rhBMP2 in mandibular carriers: expression of AP in mandibular carrier in nests of positively staining cells was seen around small and medium sized vessels, as well as, adjacent to newly-formed bone.
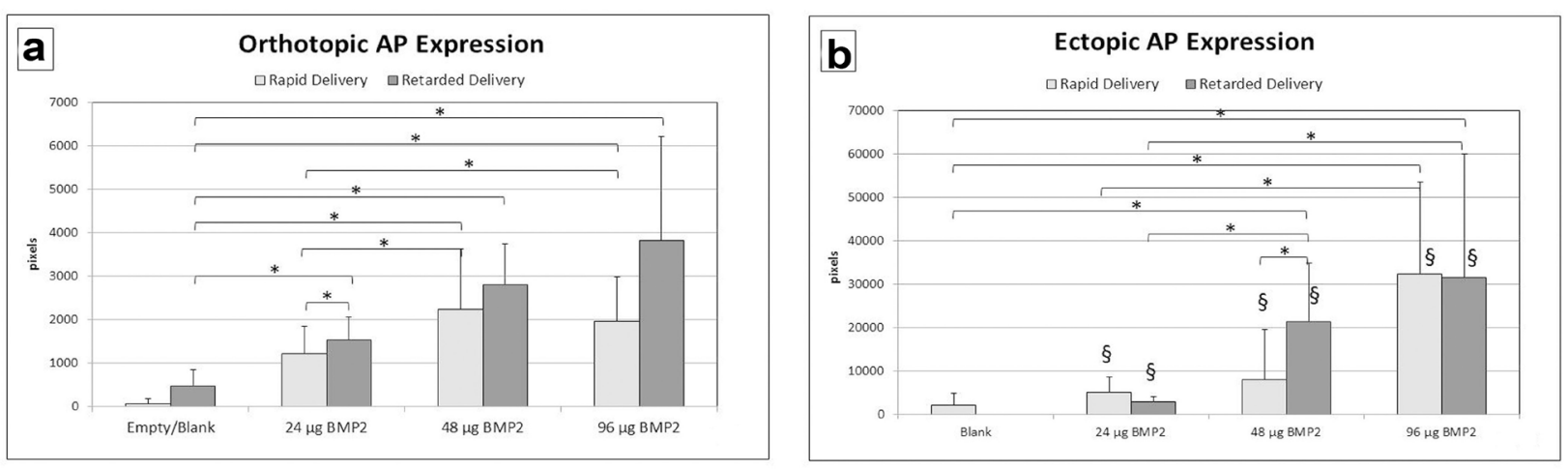

Fig. 11. Quantitative evaluation of AP expression in (a) mandibular sites and (b) ectopic sites. Comparison of paired data subsets (i.e. comparison between rapid and retarded release) was performed by paired $t$-tests. Comparison of unpaired data subsets (comparison of different dosages) was performed by ANOVA with Bonferroni correction; $n=6$; bars represent mean value, error bars represent standard deviation, ${ }^{*} p<0.05, \S=$ significantly higher expression than in orthotopic sites. 
It was particularly surprising in this regard that the anticipated delivery of very small daily amounts of rhBMP2 (between 3 and $4 \mathrm{ng}$ over a period of 21 weeks, according to the mathematical model used for curve fitting of in vitro release data) had a significant sustaining effect on bone formation in ectopic sites and coordinated the process of osteogenesis. However, when discussing the effects of individual dosages of rhBMP2 on in vivo observations, it should be kept in mind that final conclusions on the definite amounts of growth factors delivered in vivo are limited by the fact that in vivo release may differ from observed in vitro release and that mathematical fitting may not correctly reflect the real in vivo delivery per day.

Interpretation of the experimental results from a clinical point of view was of course difficult, taking into account both the size and the biology of the rodent model compared to human conditions. Nonetheless, within the limitation of this model, the results suggested that retarded delivery of small dosages of rhBMP2 in fresh bone defects of the facial skeleton could help to reduce the growth factor dosage to a certain extent. However, at the same time, it might provide no quantitative advantage, as short time high dosage regimens could have the same quantitative effect, due to a favourable regenerative environment. Hence, it seemed that the huge difference in dosage release in early periods could better address the high osteogenic potential of the fresh bone wounds, whereas the long-lasting delivery of small dosages was more effective in ectopic sides with poor osteogenic capacity.

It appears to be difficult to validate the significance of this conclusion under real current clinical conditions, where rapid delivery of largely supraphysiological dosages of BMP are used (Huh et al., 2011, de Freitas et al., 2013; Boyne et al., 2005) and dose-finding studies to reduce the amount of BMP delivered for facial bone repair are largely lacking. However, preliminary clinical results indicate that, under certain conditions, the rapid delivery of only $2-4 \%$ of the currently used dosage induces sufficient amounts of bone (Kim et al., 2014), suggesting that even rapid delivery of substantially smaller dosages can be clinically successful.

The results from ectopic sites were more difficult to transfer to a clinical scenario. Although this part of the model may resemble in an even less appropriate manner the clinical situation, results suggested that an environment with reduced osteo-regenerative characteristic may require a more controlled delivery of bone growth factors to form equivalent amounts of bone. As the majority of facial bone defects have a history of infection or previous surgical interventions, it could be advisable to use retarded delivery vehicles in these situations. If rapid release carriers were to be used, excessive dosages may be able to compensate
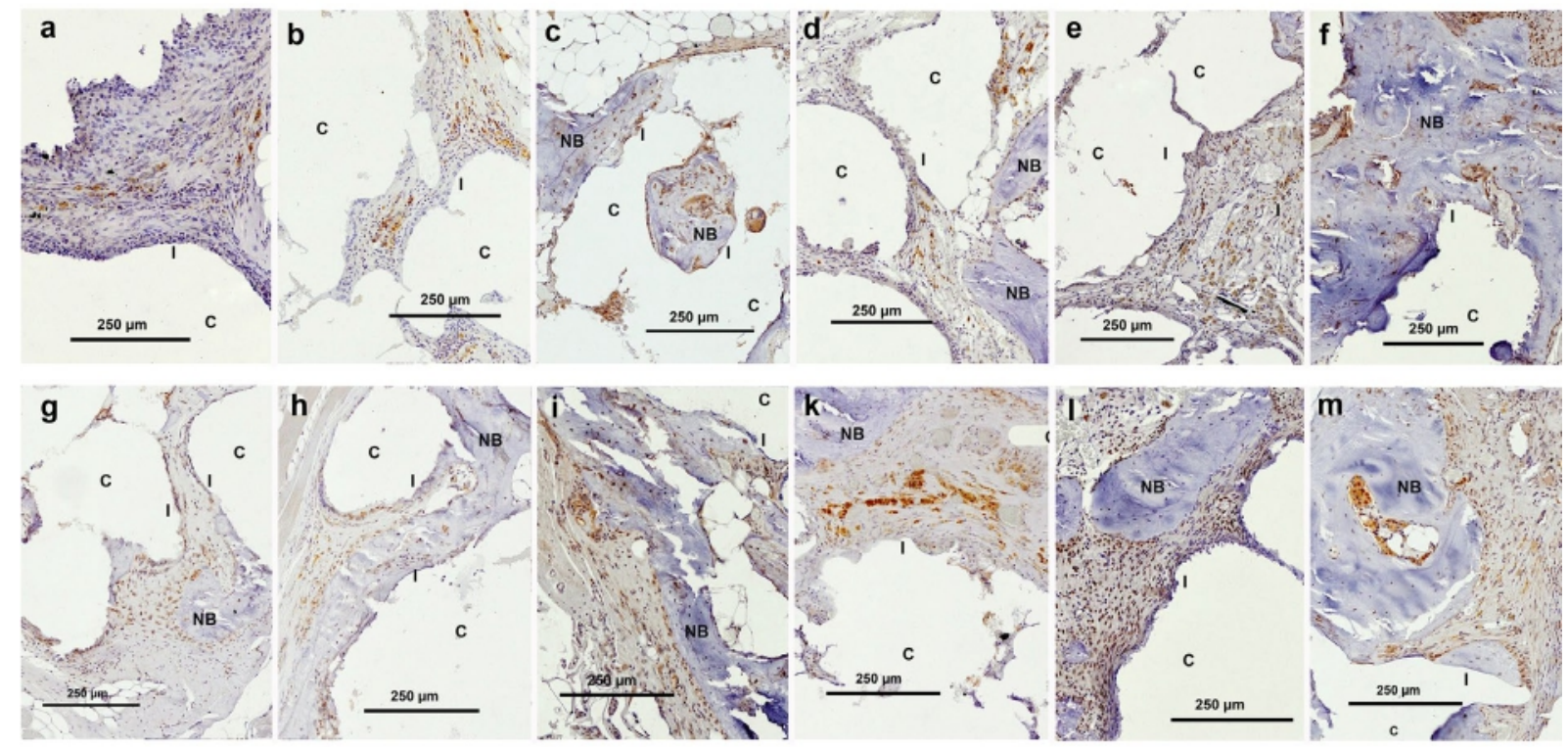

Fig. 12. Representative images of immunohistochemical features of Runx2 staining: (C) carrier material was solubilised during histologic processing, (NB) newly-formed bone, (I) interface between carrier surface and newly-formed tissue and (M) muscle tissue are indicated. Rapid delivery of (a) $24 \mu \mathrm{g}$, (b) $48 \mu \mathrm{g}$ and (c) $96 \mu \mathrm{g}$ of rhBMP2 in ectopic carriers: expression of Runx2 was observed in $(\mathbf{a}, \mathbf{b})$ both non-osseous tissue, in small groups of cells and (c) in cells adjacent to and embedded in newly-formed bone. Rapid delivery of (d) $24 \mu \mathrm{g}$, (e) $48 \mu \mathrm{g}$ and (f) $96 \mu \mathrm{g}$ of rhBMP2 in mandibular carriers: positive staining appeared to be more widespread in tissues surrounding newly-formed bone in all dosage groups. Retarded delivery (g) of $24 \mu \mathrm{g}$, (h) $48 \mu \mathrm{g}$ and (i) $96 \mu \mathrm{g}$ of rhBMP2 in ectopic carriers: similar features and distribution of expression of Runx 2 was found in ectopic carriers with retarded delivery compared to rapid delivery. Retarded delivery of (k) $24 \mu \mathrm{g}$, (1) $48 \mu \mathrm{g}$ and (m) $96 \mu \mathrm{g}$ of rhBMP2 in mandibular carriers: stronger staining for Runx 2 with more widespread occurrence was seen in mandibular carriers with retarded release when compared to carriers with rapid delivery and to ectopic carriers. 

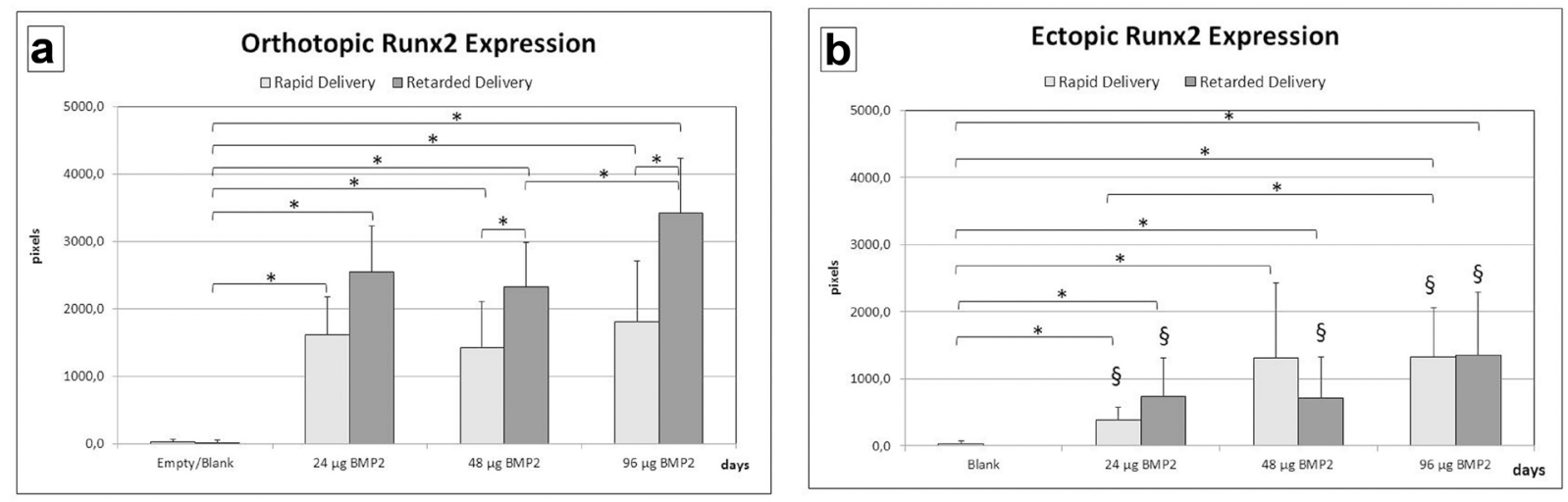

Fig. 13. Quantitative evaluation of Runx2 expression in (a) mandibular sites (b) and ectopic sites. Comparison of paired data subsets (i.e. comparison between rapid and retarded release) was performed by paired $t$-tests. Comparison of unpaired data subsets (comparison of different dosages) was performed by ANOVA with Bonferroni correction; $n=6$, bars represent mean value, error bars represent standard deviation, ${ }^{*} p<0.05, \S=$ significantly lower expression than in orthotopic sites.
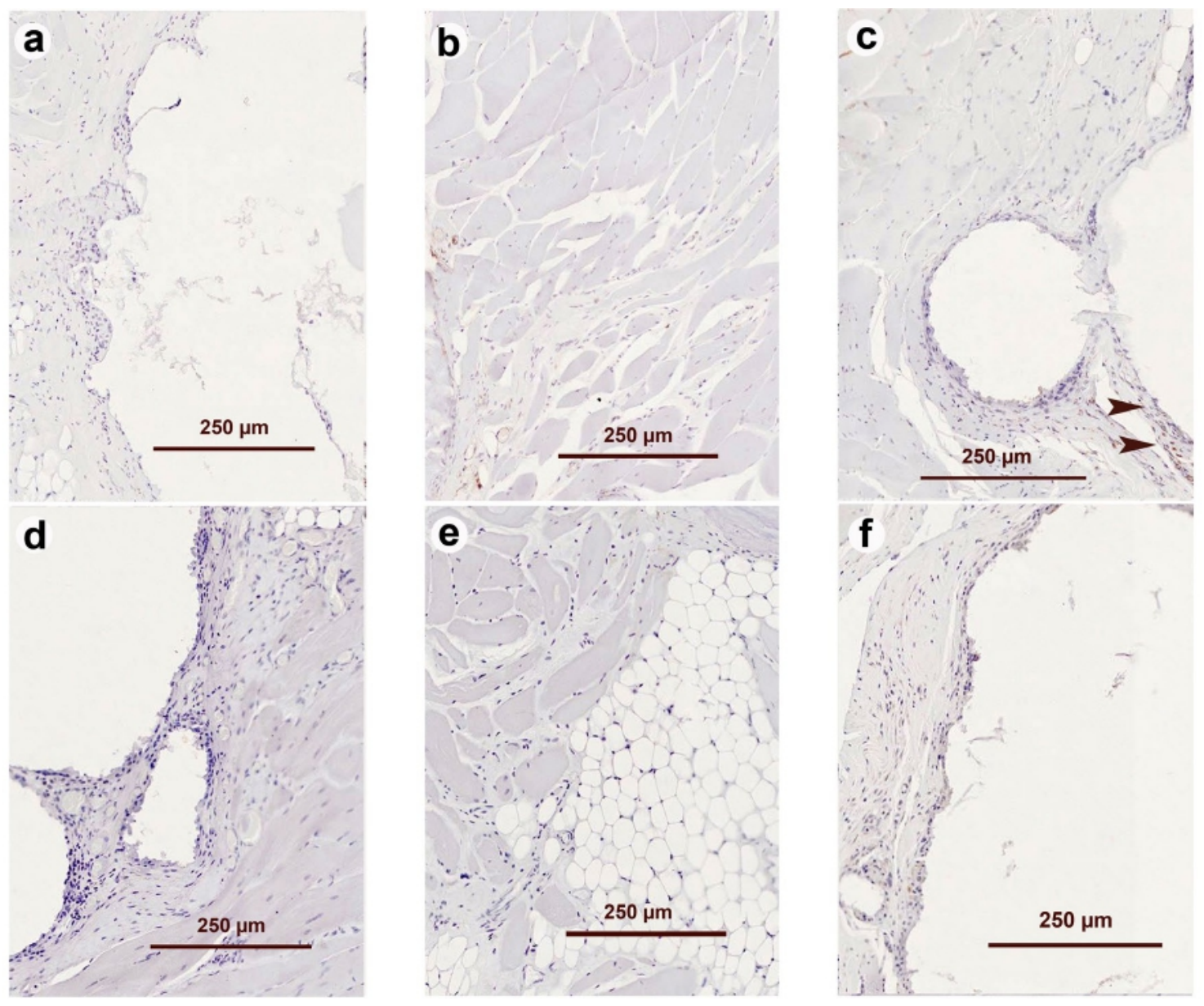

Fig. 14. AP staining of (a) blank mandibular carrier with no positive staining, (b) empty defect with muscle tissue devoid of positive staining, (c) blank ectopic carrier with weak staining occasionally visible (arrow heads). Runx2 staining of (d) blank mandibular carrier with no positive staining, (e) empty defect with muscle and fat tissue without positive staining and (f) blank ectopic carrier devoid of positive staining. 
for the inappropriate release kinetic; however, at the cost of a less effective bone formation process. The use of these supraphysiological dosages would additionally be prone to adverse events and serious side effects (Burkus et al., 2002; Smucker et al., 2006, Hwang et al., 2009).

\section{Conclusion}

Within the limitations of the experimental model and the release kinetics applied, it was concluded that retarded delivery of BMP2 was effective, preferably in sites with low or non-existing pristine osteogenic activity. At these sites, expression of bone specific markers after rapid delivery of BMP2suggested that osteogenesis might be less effective under these conditions and it might achieve equivalent bone formation only when high dosages are used.

\section{Acknowledgements}

The authors greatly value the help of Mrs. Jutta Schulz, Mrs. Ahlborn and Dr Behrens during the laboratory experiments. They wish to thank Mrs. Martha Rizk (Department of Preventive Dentistry, Periodontology and Cariology, University Medicine, Göttingen) for her support during exploration of the $\mu C T$ evaluations. They are also indebted to Dr Tim Meyer, Institute for Pharmacology and Toxicology at the University of Göttingen, for calculating the curve fittings.

This work was funded by a Grant from the Federal Ministry of Education and Research (BMBF) (No. 13N10003).

\section{References}

Boyne PJ, Lilly LC, Marx RE, Moy PK, Nevins M, Spagnoli DB, Triplett RG (2005) De novo bone induction by recombinant human bone morphogenetic protein-2 (rhBMP-2) in maxillary sinus floor augmentation. J Oral Maxillofac Surg 63: 1693-1707.

Burkus,JM, Transfeldt EE, Kitchel SH, Watkins RG, Balderston RA (2002) Clinical and radiographic outcomes of anterior lumbar interbody fusion using recombinant human bone morphogenetic protein-2. Spine 27: 2396-2408.

Buttermann GR (2008) Prospective nonrandomized comparison of an allograft with bone morphogenic protein versus an iliac-crest autograft in anterior cervical discectomy and fusion. Spine J 8: 425-435.

Carter TG, Brar PS, Tolas A, Beirne OR (2008) Offlabel use of recombinant human bone morphogenetic protein-2 (rhBMP-2) for reconstruction of mandibular bone defects in humans. J Oral Maxillofac Surg 66: 1417-1425.
Chalazonitis A, Kessler JA (2012) Pleiotropic effects of the bone morphogenetic proteins on development of the enteric nervous system. Dev Neurobiol 72: 843-856.

Clokie CM, Sándor GK (2008) Reconstruction of 10 major mandibular defects using bioimplants containing BMP-7. J Can Dent Assoc 74: 67-72.

Herford AS, Boyne PJ, Rawson R, Williams RP (2007) Bone morphogenetic protein-induced repair of the premaxillary cleft. J Oral Maxillofac Surg 65: 2136-2141.

Deckers MML, van Bezoojen RL, van der Horst G, Hoogendam J, van der Bent C, Papapoulos SE, Löwik CWGM (2002) Bone morphogenetic proteins stimulate angiogenesis through osteoblast-derived vascular endothelial growth factor A. Endocrinology 143: 1545-1553.

De Freitas RM, Spin-Neto R, Marcantonio Jun. E, Dias Pereira LAV, Wikesjö U, Susin C (2015) Alveolar ridge and maxillary sinus augmentation using rhBMP2: a systematic review. Clin Implant Dent Rel Res 17 Suppl 1: e192-201.

Delawi D, Jacobs W, van Susante JL, Rillardon L, Prestamburgo D, SPecchia N, Gay E, Verschoor N, Garcia-Fernandez C, Guerado E, Quarles van Ufford H, Kruyt MC, Dhert WJ, Oner FJ (2016) OP-1 compared with iliac crest autograft in instrumented posterolateral fusion: a randomized, multicenter noninferiority trial. J Bone Joint Surg Am 98: 441-448.

Donath K (1985) The diagnostic value of the new method for the study of undecalcified bones and teeth with attached soft tissue (Sage-Schliff (sawing and grinding) technique). Pathol Res Pract 179: 631-633.

Friedlaender GE, Perry CR, Cole JD, Cook SD, Cierny G, Muschler GF, Zych GA, Calhoun JH, LaForte AJ, Yin S (2001) Osteogenic protein-1 (bone morphogenetic protein-7) in the treatment of tibial non-unions. J Bone Joint Surg Am 83A-Suppl 1: S151158.

Garcia P, Histing T, Holstein JH, Klein M, Laschke MW, Matthys R, Ignatius A, Wildemann B, Lienau J, Peters A, Wiliie B, Duda G, Claes L, Pohlemann T; Menger MD (2013) Rodent animal models for delayed healing and non-union formation. A comprehensive review. Eur Cell Mater 26: 1-14.

Geuze RE, Theyse LF, Kempen DH, Hazewinkel HA, Kraak HY, Oner FC, Dhert WJ, Alblas J (2012) A differential effect of bone morphogenetic protein-2 and vascular endothelial growth factor release timing on osteogenesis at ectopic and orthotopic sites in a large-animal model. Tissue Eng Part A 18: 2052-62.

Glied AN, Kraut RA (2010) Off-label use of rhBMP-2 for reconstruction of critical-sized mandibular defects. N Y State Dent J 76:32-35.

Gothard D, Smith EL, Kanczler JM, Rashidi H, Qutachi O, Henstock J, Rotherham M, El Haj A, Shakesteff KM, Oreffo ROC (2014) Tissue engineered bone using select growth factors: a comprehensive review of animal studies and clinical translation studies in man. Eur Cell Mater 28: 166-208. 
Gruber RM, Weich H, Schliephake H (2009) Ectopic bone formation after implantation of a slow release system of polylactid acid and rhBMP2. Clin Oral Implants Res 20: 24-30

Gruber RM, Krohn S, Maut C, Dard M, Lange K, Perske T, Schliephake H (2014) Mandibular reconstruction using a calcium phosphate/ polyethylene glycol hydrogel carrier with BMP-2. J Clin Periodontol 41: 820-826.

Guillot R, Pignot-Paintrand I, Lavaud J, Decambron A, Bourgeois E, Josserand V, Logeart-Avramoglou D, Viguier E, Picart C (2016) Assessment of a polyelectrolyte multilayer film coating loaded with BMP-2 on titanium and PEEK implants in the rabbit femoral condyle. Acta Biomater 36: 310-22.

Herford AS, Cicciù M (2010) Recombinant human bone morphogenetic protein type 2 jaw reconstruction in patients affected by giant cell tumor. J Craniofac Surg 21: 1970-1975.

Hernandez A, Reyes R, Sanchez E, RodriguezEvora M, Delgado A, Evora C (2012) In vivo osteogenic response to different ratios of BMP-2 and VEGF released from a biodegradable porous system. J Biomed Mater Res Part A 100: 2382-2391.

Histing T, Garcia P, Holstein JH, Klein M, Matthys R, Nuetzi R, Steck R, Laschke MW, Wehner T, Bindl R, Rechnagel S, Stuermer EK, Vollmar B, Wildemann B, Lienau J, Wiliie B, Peters A, Ignatius A, Pohlemann T Claes L, Menger MD (2011) Small animal healing models: standards, tips and pitfalls resulting of a consensus meeting. Bone 49: 591-599.

Hollinger, J O, Kleinschmidt, JC (1990) The critical size defect as an experimental model to test bone repair materials. J Craniofac Surg 1: 60-68.

Huh JB, Lee HJ, Jang JW, Kim MJ, Yum PY, Kim SH, Choi KH, Kim YK, Cho KS, Shin SW (2011) Randomized clinical trial on the efficacy of Escherichia coli derived rhBMP-2 with $\beta$-TCP/HA in extraction sockets. J Adv Prosthodont 3: 161-165.

Hwang CJ, Vaccaro AR, Lawrence JP, Hong J, Schellekens H, Alaoui-Ismaili MH, Falb D (2009) Immunogenicity of bone morphogenetic proteins. J Neurosurg Spine 10: 443-451.

Jackson WM, Aragon AB, Bulken-Hoover JD, Nesti LJ, Tuan RS (2009) Putative heterotopic ossification progenitor cells derived from traumatized muscle. J Orthop Res 27: 1645-1651.

Kanczler JM, Oreffo ROC (2008) Osteogenesis and angiogenesis. Eur Cell Mater 15: 100-114.

Kelly MP, Vaughn OLA, Anderson PA (2016) Systematic review and meta-analysis of recombinant human bone morphogenetic protein-2 in localized alveolar ridge and maxillary sinus augmentation. J Oral Maxillofac Surg 74: 928-939.

Kempen DH, Lu L, Heijink A, Hefferan TE, Creemers LB, Maran A, Yaszemski MJ, Dhert WJ (2009) Effect of local sequential VEGF and BMP-2 delivery on ectopic and orthotopic bone regeneration. Biomaterials 30: 2816-25.

Kim YJ, Lee JY, Kim JE, Park JC, Shin SW, Cho KS (2014) Ridge preservation using demineralized bone matrix gel with recombinant human bone morphogenetic protein- 2 after tooth extraction: a randomized controlled clinical trial. J Oral Maxillofac Surg 72: 1281-1290.

Kopf J, Paarmann P, Hiepen J, Horbelt D, Knaus P (2014) BMP growth factor signaling in a biomechanical context. Biofactors 40: 171-187.

Lee MH; Kwon TG, Park HS, Wozney JM, Ryoo HM (2003) BMP-2 induced Osterix expression is mediated by Dlx 5 but is independent of Runx2. Biochem Biophys Res Commun 309: 689-694.

Li H, Johnson NR, Usas A, Lu A, Poddar M, Wang Y, Huard J (2013) Sustained release of bone morphogenetic protein 2 via coacervate improves the osteogenic potential of muscle-derived stem cells. Stem Cells Transl Med 2: 667-677.

Li, CJ, Madhu V, Balian G, Dighe A, Cui A (2015) Cross-talk between VEGF and BMP-6 pathways accelerates osteogenic differentiation of human adipose-derived stem cells. J Cell Physiol 230:26712682.

Matsubara T, Kida K, Yamaguchi A, Hata K, Ichida F, Meguro H, Aburatani H, Nishimura R, Yoneda T (2008) BMP2 regulates Osterix through Msx2 and Runx2 during osteoblast differentiation. J Biol Chem 283: 29119-21925.

Patel ZS, Young S, Tabata Y, Jansen JA, Wong ME, Mikos AG (2008) Dual delivery of an angiogenic and an osteogenic growth factor for bone regeneration in a critical size defect model. Bone 43: 931-40.

Rakhmatia YD, Ayukawa Y, Jinno Y, Furuhashi A, Koyano K (2017) Micro-computed tomography analysis of early stage bone healing using microporous titanium mesh for guided bone regeneration: preliminary experiment in a canine model. Odontology DOI: 10.1007/s10266-017-0298-1.

Ramazanoglu M, Lutz R, Ergun C, von Wilmowsky C, Nkenke E, Schlegel KA (2011) The effect of combined delivery of recombinant human bone morphogenetic protein-2 and recombinant human vascular endothelial growth factor 165 from biomimetic calcium-phosphate-coated implants on osseointegration. Clin Oral Implants Res 12: 1433-9.

Sacchetti B, Funari A, Remoli C, Giannicola G, Kogler G, Liedtke S, COssu G, Serafini M, Sampaolesi M, Tagliafico E, Tenedin E, Saggio I, Robey PG, Riminucci M, Bianco P (2016) No identical "mesenchymal stem cells" at different times and sites: human committed progenitors of distinct origin and differentiation potential are incorporated as adventitial cells in microvessels. Stem Cell Reports 14: 897-913.

Schliephake H; Weich H, Schulz J, Gruber H. (2007) In-vitro characterization of a slow release system of polylactic acid and rhBMP-2. J Biomed Mater Res 83: 455-462.

Schliephake H, Rublack J, Aeckerle N, Förster A, Schwenzer B, Reichert J, Scharnweber D (2015a). In vivo effect of immobilisation of bone morphogenic protein 2 on titanium implants through nanoanchored oligonucleotides. Eur Cell Mater 30: 28-40. 
Schliephake H (2015) Clinical efficacy of growth factors to enhance tissue repair in oral and maxillofacial reconstruction - a systematic review Clin Impl Dent Rel Res 17: 247-273.

Schliephake H, Vucak M, Boven J, Backhaus S, Annen T, Epple M (2015b) Solvent free production of porous PDLLA/calcium carbonate composite scaffolds improves the release of bone growth factors. Oral Maxillofac Surg 19: 133-141.

Smucker JD, Rhee JM, Singh K, Yoon T, Heller JG (2006) Increased swelling complications associated with off-label use of rhBMP-2 in the anterior cervical spine. Spine 31: 2813-2819.

Schultz RD, Bennett EE, Ellis EA, Gumienny TL (2014) Regulation of extracellular matrix organization by BMP signaling in Caenorhabditis elegans. PLoS One 11: e1011929.

Ueyama Y, Yagyuu T, Maeda M, Imada M, Akahane M, Kawate K, Tanaka Y, Kirita T (2016) Maxillofacial bone regeneration with osteogenic matrix cell sheets: an experimental study in rats. Arch Oral Biol 72: 138-145.

Yang Y, Jin G, Cao X, wang P, Yang X, Wu J (2015) In vitro evaluation of rhBMP2-induced expression of VEGF in adipose derived stromal cells. Int J Clin Exp Med 8: 222-230.

\section{Web Reference}

1. https://www.nc3rs.org.uk/arrive-guidelines.

\section{Discussion with Reviewers}

Franz Weber: The mandibular defect does not appear to be of a critical size. Would the result be different, if a critical size defect would have been chosen?

Authors: The unfilled mandibular defects regenerated only negligible amounts of bone $(\sim 0.06-0.1 \%$ of the area of bone regenerated after application of BMP2). Illustrations of blank carriers and empty defects had been omitted in order not to overload the manuscript. Representative illustrations of healing of empty defects and blank carriers (Fig. 7a-c) have been added to show that the mandibular defects were of nonhealing critical size.

Franz Weber: Bone formation will also depend on the degradation of the scaffold. Would a faster/retarded degrading scaffold yield different results?

Authors: It is quite likely that the release would have been faster if the PDLLA material had undergone a faster degradation process and hence, a faster delivery of BMP2. The difference would be likely to become visible in the ectopic locations rather than in the mandibular defects, since already the large difference in delivery of total dosage and release kinetic did not have an effect on bone formation in the mandibular sites.
Franz Weber: BMPs work excellently in rodents, but perform much worse in humans. Can these results make an impact on the clinical use of BMPs in humans?

Authors: The effect of BMPs in clinical applications in the facial skeleton has been reported to be quite predictable, if the applied excessive dosages are used in fresh bone defects (Schliephake, 2015). The results of the present study suggested that the development of retarded release devices may be one possible way to improve the performance in less favourable defect conditions. The introduction of any newly-developed material for retarded delivery into clinical use will, however, be limited by regulatory issues and the financial impact of dose-finding studies.

Georg Feichtinger: There was no benefit of retarded growth factor release apparent in the orthotopic model used. Do the authors expect a potential advantage of retarded release in an atrophic orthotopic defect? Would it be possible to reduce currently applied supraphysiological doses of BMP by choosing, in clinical settings, a retarded release approach similar to the one applied in this study?

Authors: The results suggested that a retarded release strategy did not provide an advantage in a fresh orthotopic defect. In an environment that provides a reduced level of osteogenic activity, such as atrophic defects, persistent recruitment of larger numbers of osteogenic cells will be required for effective osteogenesis. The results indicated that small amounts of BMPs, continuously delivered, may be successful in accomplishing bone formation in these situations and thereby helping to avoid supraphysiological doses.

In clinical settings, issues of regulatory approval will play a major role in the application of retarded delivery devices. In this respect, the applied PDLLA material and the solvent-free process of growth factor incorporation may be less critical for a retarded delivery approach in clinical use. The transfer from a rodent model into a clinical application would, however, still require a substantial effort in the direction of dose-finding studies.

Georg Feichtinger: You stated that ectopic intramuscular implantation might represent atrophic defects, where no potent osteogenic precursors are available within the vicinity of the defect. However, there is excellent perfusion and soft tissue support available in the chosen intramuscular ectopic model. How does this influence osteogenic capacity? Are there any other ectopic models available, which would provide a better representation of atrophic situations - if at all possible?

Authors: We agree that ectopic sites do not ideally reflect the situation of atrophic defects, as vascularity is unaffected and soft tissue quality is not deteriorated due to previous surgery or trauma. However, it is interesting to note that in mice non-union fracture 
models bone formation failed and atrophic nonunions formed despite increased vascularisation (Garcia et al., 2012; additional reference). The results of that study indicated that vascularity as such may not be a crucial factor in the enlarged osteotomy gaps, but rather a disturbed ratio of angiogenic to osteogenic growth factors. If we transfer these insights to the idea of ectopic sites reflecting an atrophic defect situation, we could conclude that unimpaired vascularisation in ectopic sites may not contradict this idea. It may be that the absence of pristine osteogenic cells (for example in the enlarged gap osteotomies in the cited study) is a more important feature that accounts for the disturbed ratio of growth factors, thereby leading to an atrophic defect situation. Nevertheless, one could imagine that in subcutaneous sites, recruitment of mesenchymal precursor cells for osteogenic differentiation may be even more challenging. In this way, these sites would be interesting to be explored for their capacity to form bone, using retarded release of osteogenic growth factors.

Arie Bruinink: Comparing the amount of BMP2 that was released per day under rapid and retarded release conditions, it seems that only in the period 0-7 d the difference in released BMP2 amount was tremendous. In the period of 7 to $35 \mathrm{~d}$, the released amount was in a similar range. Regarding the outcome, how important was the 7-35 d release period?

Authors: It is true that the difference between the two release regimens was larger during the period 0-7 $\mathrm{d}$ and in a similar range thereafter, until day 35 . However, the calculations of the release beyond this point - based on the data of day 0-35 and assuming that in vitro release represented in vivo delivery - indicated that the short release of high dosages of BMP2 followed a simple exponential function that was expected to provide no further release after 5 weeks, whereas the slow small dosage delivery exhibited a double exponential function that was supposed to continue release over another 21 weeks. In orthotopic sites, this continued release of small dosages of BMP2 appeared to save dosage, but provided no advantage with regard to the extent of bone formation, where rapid delivery of higher dosages could accomplish the same effect. In contrast, in ectopic sites, devoid of pristine osteogenic activity, the slow small dosage regimen with a continued release over 6 months was more efficient and led to larger amounts of bone, when compared to rapid delivery of high and medium dosages of BMP2. Hence, it appeared that the huge difference in dosage release in early periods could better address the high osteogenic potential of the fresh bone wounds, whereas the long-lasting delivery of small dosages was more effective in ectopic sides with poor osteogenic capacity.

\section{Additional reference}

Garcia P, Pieruschka A, Klein M, Tami A, Histing T, Holstein JH, Scheuer C, Pohlemann T, Menger MD (2012) Temporal and spatial vascularization patterns of unions and nonunions: role of vascular endothelial growth factor and bone morphogenetic proteins. J Bone Joint Surg Am 94: 49-58.

Editor note: The scientific editor for this paper was Christine Hartmann. 